\title{
Irreducible cycles and points in special position in moduli spaces for tropical curves
}

\author{
Andreas Gathmann \\ Fachbereich Mathematik \\ TU Kaiserslautern \\ Postfach 3049, 67653 Kaiserslautern, Germany \\ andreas@mathematik.uni-kl.de \\ Franziska Schroeter \\ Cluster of Excellence for Computer Science \\ Universität des Saarlandes \\ Postfach 151150, 66041 Saarbrücken, Germany \\ schroeter@math.uni-sb.de
}

Submitted: Dec 8, 2011; Accepted: Oct 31, 2012; Published: Nov 15, 2012

Mathematics Subject Classifications: 14N10, 14T05

\begin{abstract}
In the first part of this paper, we discuss the notion of irreducibility of cycles in the moduli spaces of $n$-marked rational tropical curves. We prove that Psiclasses and vital divisors are irreducible, and that locally irreducible divisors are also globally irreducible for $n \leqslant 6$. In the second part of the paper, we show that the locus of point configurations in $\left(\mathbb{R}^{2}\right)^{n}$ in special position for counting rational plane curves (defined in two different ways) can be given the structure a tropical cycle of codimension 1 . In addition, we compute explicitly the weights of this cycle.
\end{abstract}

\section{Introduction}

In classical algebraic geometry, a divisor on a nonsingular algebraic variety $X$ over $\mathbb{C}$ is a (formal) finite $\mathbb{Z}$-linear combination of irreducible subvarieties of codimension 1 . Interpreting a divisor as an algebraic variety (with multiplicities attached to its components) it is of course irreducible if and only if it consists of only one subvariety. In tropical geometry however, we define a divisor in a tropical fan $X$ to be, roughly speaking, a weighted subfan of codimension one - so in the case of non-negative weights it is just an embedded tropical variety. As there is no unique decomposition of a such a variety into irreducible components (see Remark 2.18), the situation is much more complicated here. The notion of irreducible tropical fan was introduced in $[\mathrm{GKM}]$ and can easily be generalized 
to tropical divisors in $X$ (Definition 2.17). A divisor is then irreducible if and only if its weight function is unique up to a global multiple (Lemma 2.20). We explore this notion when the ambient space is $X=\mathcal{M}_{0, n}$, the parameter space of tropical rational $n$-marked tropical curves [GKM, M], or equivalently the tropical Grassmannian $\mathcal{G}(2, n)$ modulo its lineality space [SS]. We prove for example that tropical Psi-classes and vital divisors are irreducible. Note that the corresponding statement is also true in algebraic geometry for the vital divisors $[\mathrm{K}]$, where they form the main objects in the intersection theory on the moduli spaces $\bar{M}_{0, n}$ of stable curves. In contrast, Psi-classes in algebraic geometry cannot be defined as divisors, but only as divisor classes on $\bar{M}_{0, n}[\mathrm{AC}]$, and hence the notion of irreducibility does not make sense for them. They are useful for the construction of enumerative invariants like descendant Gromov-Witten numbers as in [KoM]. Furthermore we show that for $n \leqslant 6$ every divisor in $\mathcal{M}_{0, n}$ that is locally irreducible is already globally irreducible (Proposition 2.36).

The second part of this paper, which is almost independent of the first, deals with the tropical notion of points in special position for enumerative problems. If one wants to count for instance curves of degree $d$ in $\mathbb{P}^{2}$ passing through $n=3 d-1$ given points, these point conditions should be generic enough to obtain at least a finite result that is independent of the conditions. We call the set of these generic point configurations in $\left(\mathbb{P}^{2}\right)^{n}$ the set of points in general position. There exist several definitions of this notion we can for example just require the number of curves to be finite, or in addition that the curves are all smooth. A common feature of these definitions is that they lead to an open dense subset of $\left(\mathbb{P}^{2}\right)^{n}$ of points in general position. The complement of this set in $\left(\mathbb{P}^{2}\right)^{n}$ is called the set of points in special position. We explore this notion in the corresponding tropical context. It turns out that this set is the support of a tropical fan in $\left(\mathbb{R}^{2}\right)^{n}$, more precisely of a push-forward of a divisor in the tropical moduli space corresponding

to the classical moduli space $\overline{\mathcal{M}}_{0,3 d-1}\left(\mathbb{P}^{2}, d\right)$ of stable maps (Propositions 3.10 and 3.14). Finally, we compute the weights of the top-dimensional cones in this tropical fan by means of elementary divisors of certain matrices (Corollary 3.30).

We would like to thank Hannah Markwig for inspiring and helpful discussions. Part of this work was done at the Mathematical Sciences Research Institute (MSRI) in Berkeley, CA, USA, during the one-semester program on tropical geometry in fall 2009. The authors would like to thank the MSRI for hospitality and support. In particular, Andreas Gathmann was supported by the Simons Professorship of the MSRI.

\section{2 (Irreducible) tropical subfans and cycles in $\mathcal{M}_{0, n}$}

In this section, we want to verify that the codimension- $k$ skeletons of the moduli spaces $\mathcal{M}_{0, n}$ of rational $n$-marked abstract tropical curves are tropical subvarieties, for suitable weight functions. Furthermore, it turns out that the same holds for codimension- $k$ skeletons of Psi-classes $\psi_{i}$ living in $\mathcal{M}_{0, n}$. This will be done by checking the balancing condition explicitly in terms of coordinate vectors of $\mathcal{M}_{0, n}$. Inspired by this method, we will show in the subsequent part that tropical Psi-classes $\psi_{i}$ are irreducible. In addition, we will prove that the tropical vital divisors $D^{S}$ in $\mathcal{M}_{0, n}$ are also irreducible. As a by-product we 
will give a new characterization of irreducible tropical fans and cycles.

Let us start by reviewing the definitions and notions indispensable below and by fixing the notation. More details about the following notions can be found in [GKM, Definitions 2.4, 2.6. and 2.8].

Definition 2.1 (Tropical (sub-)fans, codimension- $k$ skeleton). Pick some $r \geqslant 0$ and a lattice $\Lambda \cong \mathbb{Z}^{r}$; set $V:=\Lambda \otimes_{\mathbb{Z}} \mathbb{R}$.

1. Let $X$ be a fan of pure dimension $N$ in $V$. For $0 \leqslant k \leqslant N$ we denote the collection of its $k$-dimensional cones by $X^{(k)}$. The vector subspace of $V$ spanned by a cone $\sigma$ of $X$ will be denoted $V_{\sigma}$. We call $X$ a tropical fan if it is equipped with a weight function $w: X^{(N)} \rightarrow \mathbb{N}$ such that the balancing condition

$$
\sum_{\sigma>\tau} w(\sigma) v_{\sigma / \tau}=0 \quad \in V / V_{\tau}
$$

holds for all $(N-1)$-dimensional cones $\tau$, where $v_{\sigma / \tau}$ is the primitive normal vector of $\sigma$ relative to $\tau$. The union of all cones of $X$ will be written as $|X| \subset V$.

2. A (tropical) subfan $Y$ of a (tropical) fan $X$ in $V$ is itself a (tropical) fan $Y$ in $V$ with the property that each cone of $Y$ is contained in a cone of $X$. Note that the weight function of $Y$ is not necessarily inherited by $X$.

3. The codimension- $k$ skeleton $X_{\mathrm{sk}}^{(N-k)}$ of a fan $X$ in $V$ of pure dimension $N$ (for $0 \leqslant k \leqslant N)$ consists of all cones of dimension at most $N-k$ in $X$. It is a puredimensional fan of dimension $N-k$, however with no canonical weight function associated to it.

Construction 2.2 (Moduli space $\mathcal{M}_{0, n}$ of rational $n$-marked abstract tropical curves). A rational $n$-marked (abstract) tropical curve is a tuple $\left(\Gamma ; x_{1}, \ldots, x_{n}\right)$, where $\Gamma$ is a connected metric tree graph all of whose vertices have valence at least 3 , and where $x_{1}, \ldots, x_{n}$ is a labeling of its unbounded edges. In the following, we will sometimes write marked edge $x_{i}$ or marked end $x_{i}$ for the unbounded edge with label $x_{i}$. An isomorphism of rational tropical curves $\left(\Gamma ; x_{1}, \ldots, x_{n}\right)$ and $\left(\tilde{\Gamma} ; \tilde{x}_{1}, \ldots, \tilde{x}_{n}\right)$ is a homeomorphism $\Gamma \rightarrow \tilde{\Gamma}$ sending $x_{i}$ to $\tilde{x}_{i}$ and mapping the edges of $\Gamma$ bijectively onto edges of $\tilde{\Gamma}$ by affine maps of slope \pm 1 . As in $[\mathrm{GKM}]$, we will denote by $\mathcal{M}_{0, n}$ the parameter space of all such curves modulo isomorphisms. For many purposes it plays the tropical role of the classical moduli space of rational $n$-marked stable curves - even if it is not compact.

The moduli space $\mathcal{M}_{0, n}$ can be given the structure of a tropical fan (see Remark 2.3

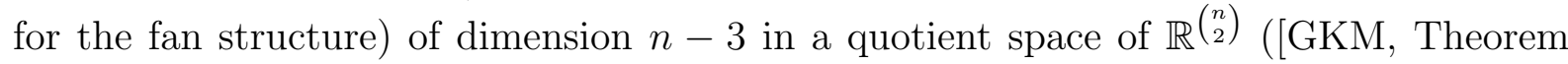
$3.7])$; in fact, it can be described as the tropical Grassmannian $\mathcal{G}(2, n)$ modulo its lineality space (see [SS, Theorem 3.4] or [GKM, Remark 3.9]). More precisely, for each $n$-marked tropical curve $\left(\Gamma ; x_{1}, \ldots, x_{n}\right)$ and $1 \leqslant i<j \leqslant n$ let $\operatorname{dist}_{\Gamma}\left(x_{i}, x_{j}\right)$ be the distance between the unbounded edges $x_{i}$ and $x_{j}$ in $\Gamma$. We thus get a map

$$
\begin{aligned}
\tilde{v}: \quad \mathcal{M}_{0, n} & \rightarrow \mathbb{R}^{\left(\begin{array}{l}
n \\
2
\end{array}\right)} \\
\left(\Gamma ; x_{1}, \ldots, x_{n}\right) & \mapsto\left(\operatorname{dist}_{\Gamma}\left(x_{i}, x_{j}\right)\right)_{i<j}
\end{aligned}
$$




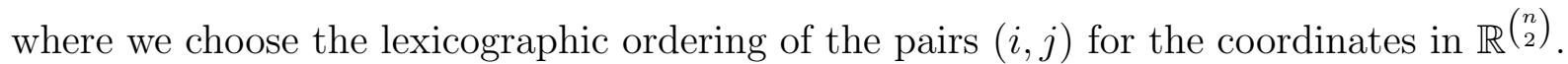
We will call $\tilde{v}\left(\Gamma ; x_{1}, \ldots, x_{n}\right)$ the distance vector of $\left(\Gamma ; x_{1}, \ldots, x_{n}\right)$.

The following vectors in $\mathbb{R}^{\left(\begin{array}{l}n \\ 2\end{array}\right)}$ will be of particular importance: let $I \subset\{1, \ldots, n\}$ be

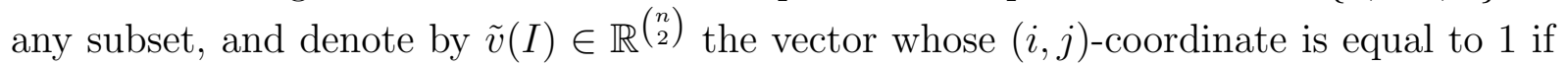
$I$ contains exactly one of the numbers $i$ and $j$, and 0 otherwise. Note that $\tilde{v}\left(I^{c}\right)=\tilde{v}(I)$, where $I^{c}$ denotes the complement of $I$ in $\{1, \ldots, n\}$.

The vectors $d_{i}:=\tilde{v}(\{i\})$ for $i=1, \ldots, n$ form a basis of the so-called lineality space mentioned above; i.e. by taking the quotient by this subspace we obtain a map

$$
\begin{aligned}
v: \quad \mathcal{M}_{0, n} & \rightarrow \mathbb{R}^{\left(\begin{array}{c}
n \\
2
\end{array}\right)} /\left\langle d_{1}, \ldots, d_{n}\right\rangle \\
\left(\Gamma ; x_{1}, \ldots, x_{n}\right) & \mapsto \overline{\left(\operatorname{dist}_{\Gamma}\left(x_{i}, x_{j}\right)\right)_{i<j}}
\end{aligned}
$$

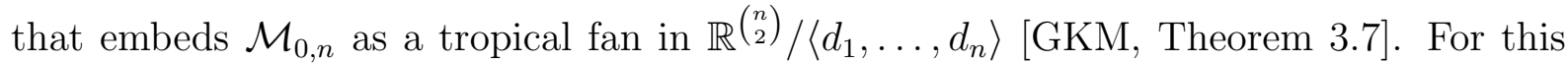
structure of a tropical fan the weights of all top-dimensional cones are chosen to be 1 , and the underlying lattice $\Lambda$ is taken to be the one generated by the classes $v(I)$ of the vectors $\tilde{v}(I)$ for all $I \subset\{1, \ldots, n\}$ modulo the lineality space. In the remainder, we will always view $\mathcal{M}_{0, n}$ as a tropical fan with this embedding.

Remark 2.3. The cones of the tropical fan $\mathcal{M}_{0, n}$ correspond to curves of the same combinatorial type, i.e. curves which differ just by the lengths of their bounded edges. They are open convex polyhedra of dimension

$$
n-3-\sum_{\text {vertices } V \text { in } \Gamma}(\text { val } V-3),
$$

where val $V$ is the valence of the vertex $V$ of any curve $\Gamma$ of the given type. The onedimensional cones of $\mathcal{M}_{0, n}$ are generated by the vectors $v(I)$ of Construction 2.2 for all $I \subset\{1, \ldots, n\}$ with $2 \leqslant|I| \leqslant n-2$; by construction these are just the distance vectors of curves $\left(\Gamma ; x_{1}, \ldots, x_{n}\right)$ in $\mathcal{M}_{0, n}$ having exactly one bounded edge of length 1 , with the markings $I$ to one and $I^{c}$ to the other side. Hence there is only a finite number of combinatorial types of curves in $\mathcal{M}_{0, n}$. Note that the fan structure of $\mathcal{M}_{0, n}$ as in Construction 2.2 coincides with the fan structure of the quotient of $\mathcal{G}(2, n)$ by its lineality space, see [SS, Theorem 4.2 and proof of Theorem 3.4].

Definition and Remark 2.4 (Notions of intersection theory for our purpose, see [AR] for more details). A tropical (affine) $k$-cycle is a weighted fan of pure dimension $k$ in $V$ satisfying the balancing condition but where the range of the weight function is $\mathbb{Z}$. Hence the difference between a tropical fan of dimension $k$ and a tropical $k$-cycle is just the range of the weight function. A tropical $k$-cycle in a tropical fan $X$ is simply a $k$-cycle that is a subfan of $X$. Typically a tropical $k$-cycle in $X$ is an equivalence class of weighted fans of pure dimension $k$, where two fans are equivalent if they have a common refinement [AR, Definition 2.8]. However, in this paper we will not distinguish between equivalence classes and representatives of the equivalence class as the difference does not concern us here.

As before, we will denote by $|X|$ the subset of $V$ of all cones of $X$ (with non-zero weight). A (Weil) divisor $D$ on $X$ is a cycle in $X$ of codimension 1 . 
Let $X$ be a $k$-cycle. A (non-zero) rational function on $X$ is a continuous function $f:|X| \rightarrow \mathbb{R}$ which is integer affine linear on each cone $\sigma \subset|X|$. We denote the linear part of the restriction of $f$ to $\sigma$ by $f_{\sigma}$.

Let $X$ be a $k$-cycle and $f:|X| \rightarrow \mathbb{R}$ a rational function on $X$. The Weil divisor $D(f)$ associated to $f$ is the divisor in $X$ consisting of the codimension- 1 cones $\tau \in X^{(k-1)}$ with weights

$$
w(\tau)=\sum_{\sigma>\tau} w(\sigma) f_{\sigma}\left(v_{\sigma / \tau}\right)-f_{\tau}\left(\sum_{\sigma>\tau} w(\sigma) v_{\sigma / \tau}\right),
$$

where the sum runs over all cones $\sigma$ s.t. $\tau \subsetneq \sigma$ is a face of $\sigma$, and $v_{\sigma / \tau}$ denotes the primitive normal vector of $\sigma$ relative to $\tau$. It has been shown in [AR, Proposition 3.7] that this is indeed a tropical cycle.

Definition and Remark 2.5 (Tropical Psi-classes $\psi_{i}$ ). Fix $n>2$ and $i \in\{1, \ldots, n\}$. The Psi-class $\psi_{i} \subset \mathcal{M}_{0, n}$ is the subfan of $\mathcal{M}_{0, n}$ consisting of all cones of $\mathcal{M}_{0, n}$ of curves $\left(\Gamma ; x_{1}, \ldots, x_{n}\right)$ such that the marked edge $x_{i}$ is adjacent to a vertex of valence at least $4[\mathrm{M}]$. Giving each top-dimensional cone the weight 1 it has the structure of a tropical subfan of $\mathcal{M}_{0, n}$ of codimension 1. Using the language of [AR], we can rephrase this as: $\psi_{i}$ is a tropical Weil divisor associated to a rational function as proven in [KM, Proposition 3.5]. Note that a Psi-class is not defined up to rational equivalence as it is the case in classical geometry (and should therefore better be named Psi-divisor).

Convention 2.6. When we talk in the following of "the" codimension- $k$ skeleton of $\mathcal{M}_{0, n}$ respectively $\psi_{i}$, we mean this w.r.t. the fan structure of Remarks 2.3 and 2.5.

Remark 2.7 (Vital divisors). Going back to Sean Keel [K], in the classical world the vital divisors generate the divisor class group of the moduli spaces $\bar{M}_{0, n}$ of rational $n$-marked stable curves and are sometimes called boundary divisors of $\bar{M}_{0, n}$. The tropical analogue has first been studied by Matthias Herold in his diploma thesis $[\mathrm{H}]$. The results are also partly mentioned in [R, Definition 2.4 and Lemma 2.5]. We will introduce them here carefully.

Definition 2.8 (Tropical vital divisors in $\mathcal{M}_{0, n}$ ). Let $n \geqslant 4$ and $S \subset\{1, \ldots, n\}$ with $2 \leqslant|S| \leqslant n-2$. As $\mathcal{M}_{0, n}$ is a simplicial fan with rays generated by the vectors $v(I)$ of Remark 2.3 there is a unique piecewise linear function $\phi_{S}$ on $\mathcal{M}_{0, n}$ linear on each cone that takes the value 1 on the vector $v(S)$ and 0 on all other rays. The associated Weil divisor $D\left(\phi_{S}\right)$ to this function is called vital divisor w.r.t. $S$ and is denoted by $D^{S}$.

As $v(S)=v\left(S^{c}\right)$ it is clear from the definition that $D^{S}=D^{S^{c}}$. Moreover, the following proposition taken from [H, proof of Theorem 3.7] computes the weight function of the divisor $D^{S}$ explicitly:

Proposition 2.9 (Weight functions of the vital divisors). Let $n \geqslant 4$ and $S \subset\{1, \ldots, n\}$ with $2 \leqslant|S| \leqslant n-2$. Let $\tau \in \mathcal{M}_{0, n}^{(n-4)}$ be a cone of codimension 1; by Remark 2.3 its corresponding curves have exactly one 4 -valent vertex. Denote by $A, B, C, D \subset\{1, \ldots, n\}$ 
the sets of marked ends behind each of the four edges adjacent to this vertex. Then the weight of the vital divisor $D^{S}$ on the cone $\tau$ is

$$
w(\tau)= \begin{cases}1 & \text { if } S=I \cup J, \text { where } I \neq J \text { and } I, J \in\{A, B, C, D\} \\ -1 & \text { if } S=I \text { or } S^{c}=I \text { with } I \in\{A, B, C, D\} \\ 0 & \text { otherwise }\end{cases}
$$

Proof. Adjacent to our given codimension-1 cone $\tau$ of $\mathcal{M}_{0, n}$ there are three top-dimensional cones $\sigma_{1}, \sigma_{2}, \sigma_{3}$ corresponding to resolving the 4 -valent vertex in the three ways shown in the picture below.
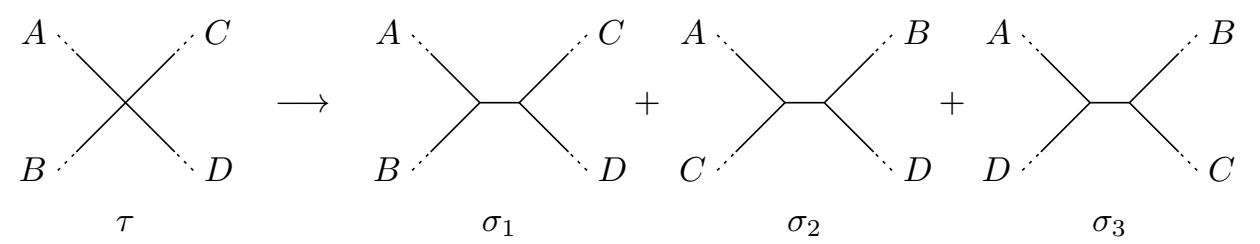

The primitive normal vectors $v_{\sigma_{i} / \tau}$ for $i=1,2,3$ as in Remark 2.4 are then just the distance vectors of the three curves shown above on the right, with the newly added edge having length 1 and all other edges shrunken to zero, i.e. we have

$$
v_{\sigma_{1} / \tau}=v(A \cup B), \quad v_{\sigma_{2} / \tau}=v(A \cup C), \text { and } \quad v_{\sigma_{3} / \tau}=v(A \cup D)
$$

with the vectors $v(\cdot)$ as defined in Remark 2.3. As $\mathcal{M}_{0, n}$ is balanced [GKM, proof of Theorem 3.7] we know moreover that

$$
v(A \cup B)+v(A \cup C)+v(A \cup D)=v(A)+v(B)+v(C)+v(D) .
$$

Using Definition 2.8 of the function $\phi_{S}$, an application of the formula of Definition 2.4 for the weight $w(\tau)$ of the divisor $D^{S}=D\left(\phi_{S}\right)$ on $\tau$ thus yields (note that the weights of $\mathcal{M}_{0, n}$ are all 1$)$

$$
\begin{aligned}
w(\tau) & =\sum_{i=1}^{3}\left(\phi_{S}\right)_{\sigma_{i}}\left(v_{\sigma_{i} / \tau}\right)-\left(\phi_{S}\right)_{\tau}\left(\sum_{i=1}^{3} v_{\sigma_{i} / \tau}\right) \\
= & \phi_{S}(v(A \cup B))+\phi_{S}(v(A \cup C))+\phi_{S}(v(A \cup D))-\phi_{S}(v(A)+v(B)+v(C)+v(D)) \\
= & \begin{cases}1 & \text { if } S=I \cup J \text { with } I \neq J \text { and } I, J \in\{A, B, C, D\}, \\
-1 & \text { if } S=I \text { or } S^{c}=I \text { with } I \in\{A, B, C, D\}, \\
0 & \text { otherwise. }\end{cases}
\end{aligned}
$$

Example 2.10 (Vital divisors in $\mathcal{M}_{0,5}$ ). By symmetry, in $\mathcal{M}_{0,5}$ we just have to consider sets $S$ with $|S|=2$. There are 10 such sets and thus 10 vital divisors. Set for example $S=\{1,2\}$. Curves of codimension 1 in $\mathcal{M}_{0,5}$ have one bounded edge on exactly one of whose ends lies a 4 -valent vertex. So in the notation of Proposition 2.9 we have w.l.o.g. $|A|=2 ; A$ determines the type of the curve $\mathcal{M}_{0,5}$ uniquely. Using the weight function above, we get the following weights: 


\begin{tabular}{|c|cccccccccc|}
\hline$A=$ & $\{1,2\}$ & $\{1,3\}$ & $\{1,4\}$ & $\{1,5\}$ & $\{2,3\}$ & $\{2,4\}$ & $\{2,5\}$ & $\{3,4\}$ & $\{3,5\}$ & $\{4,5\}$ \\
\hline weight & -1 & 0 & 0 & 0 & 0 & 0 & 0 & 1 & 1 & 1 \\
\hline
\end{tabular}

We now want to check that codimension- $k$ skeletons of and Psi-classes in $\mathcal{M}_{0, n}$ are in fact tropical fans, i.e. that they satisfy the balancing condition. So let us fix $k \in$ $\{0, \ldots, n-3\}$ and a weight function $w: \mathcal{M}_{0, n}^{(n-3-k)} \rightarrow \mathbb{Z}_{>0}$ on the codimension- $k$ skeleton of $\mathcal{M}_{0, n}$. Moreover, let $\tau \in \mathcal{M}_{0, n}^{(n-3-k-1)}$ be a cone, corresponding by Remark 2.3 to a certain combinatorial type of $n$-marked curves. In order to verify the balancing condition for $w$ at $\tau$, the following toolkit will be useful.

Remark 2.11 (About the balancing condition in $\mathcal{M}_{0, n}$ ). The cones $\sigma \in \mathcal{M}_{0, n}^{(n-3-k)}$ containing $\tau$ can be obtained by resolving one chosen vertex $V$ of valence $r$ at least 4 in $\tau$ in the same way as it can be resolved in $\mathcal{M}_{0, r}$ by adding one bounded edge. More precisely, this means that we replace $V$ by two vertices joined by a bounded edge, with the $r$ edges of $V$ split up in every possible way onto the two new vertices such that there are at least two such edges on each side of the bounded edge. The following picture shows these types of resolutions; it can either be seen as a picture of curves in $\mathcal{M}_{0, r}$ or as a local picture of curves in $\mathcal{M}_{0, n}$ around $V$.

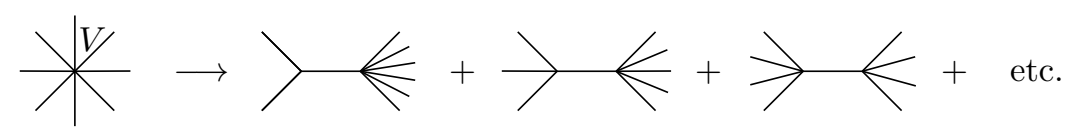

In order to check the balancing condition at $\tau$ it therefore suffices to split the total sum $\sum_{\sigma>\tau} w(\sigma) v_{\sigma / \tau}$ into parts, where each part corresponds to the resolution of one vertex $V$, and verify it for each part separately. This leads to the following lemma which states that the balancing condition for codimension- $k$ cells in $\mathcal{M}_{0, n}$ can be split up into several conditions in lower-dimensional moduli spaces.

Lemma 2.12 (Splitting the balancing condition). With notations being as above, let $\left\{V_{1}, \ldots, V_{m}\right\}$ be the set of 4 - or higher valent vertices of a curve in $\tau$, and let $r_{1}, \ldots, r_{m}$ be their respective valences. Then to verify the balancing condition at $\tau$ in $\mathcal{M}_{0, n}$ it suffices to check the balancing at all 0-dimensional cones $\tau_{i}$ in $\mathcal{M}_{0, r_{i}}$ for $i \in\{1, \ldots, m\}$ (corresponding to curves ("stars") having only one vertex of valence $r_{i}$ ).

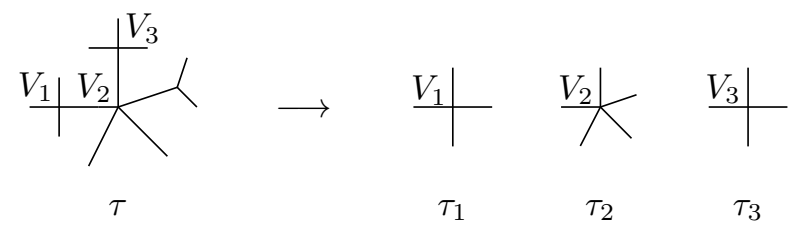

Proof. Consider a 4- or higher valent vertex $V \in\left\{V_{1}, \ldots, V_{m}\right\}$ of $\tau$, let $r$ be its valence, and denote by $A_{i}$ for $i=1, \ldots, r$ the set of marked edges behind the $i$-th edge of $V$. Thus we have $\sqcup_{i} A_{i}=\{1, \ldots, n\}$. Define a linear map $\phi$ by

$$
\phi: \mathbb{R}^{\left(\begin{array}{l}
r \\
2
\end{array}\right)} \rightarrow \mathbb{R}^{\left(\begin{array}{l}
n \\
2
\end{array}\right)}, \quad\left(x_{i, j}\right)_{i<j} \mapsto\left(\tilde{x}_{k, l}\right)_{k<l}
$$


where

$$
\tilde{x}_{k, l}= \begin{cases}x_{i, j} & \text { if }(k, l) \in A_{i} \times A_{j} \text { or }(l, k) \in A_{i} \times A_{j} \text { for some } 1 \leqslant i<j \leqslant r, \\ 0 & \text { otherwise }\end{cases}
$$

for $1 \leqslant k<l \leqslant n$. Then by construction the distance vectors of Construction 2.2 are transformed by $\phi$ as

$$
\phi(\tilde{v}(I))=\tilde{v}\left(\bigcup_{i \in I} A_{i}\right)
$$

for all subsets $I \subset\{1, \ldots, r\}$. In particular, for $i=1, \ldots, r$ the $i$-th basis vector $d_{i}$ of the lineality space of $\mathcal{M}_{0, r}$ is mapped to $\phi\left(d_{i}\right)=\tilde{v}\left(A_{i}\right)$.

Let $\tau_{\star}$ be the 0 -dimensional cone in $\mathcal{M}_{0, r}$, corresponding to the star curve with one vertex. By Remark 2.11 a 1-dimensional cone $\sigma_{\star}$ around $\tau_{\star}$ corresponds to a cone $\sigma$ around $\tau$ in $\mathcal{M}_{0, n}$ describing the same local resolution. Let us assume that the balancing condition holds at $\tau_{\star}$, i.e. that

$$
\sum_{\sigma_{\star}>\tau_{\star}} w(\sigma) \tilde{v}_{\sigma_{\star} / \tau_{\star}}=\sum_{i} a_{i} d_{i} \quad \in \mathbb{R}^{\left(\begin{array}{c}
r \\
2
\end{array}\right)}
$$

for some $a_{i} \in \mathbb{R}$. By Remark 2.3 the normal vectors $\tilde{v}_{\sigma_{\star} / \tau_{\star}}$ in $\mathcal{M}_{0, r}$ are exactly $\tilde{v}(I)$ for the corresponding subset $I \subset\{1, \ldots, r\}$ with $2 \leqslant|I| \leqslant r-2$, and by (1) these vectors are mapped by $\phi$ to the corresponding normal vectors $\tilde{v}_{\sigma / \tau}$ in $\mathcal{M}_{0, n}$. So applying $\phi$ to (2) we get

$$
\sum_{\sigma_{*}>\tau_{*}} w(\sigma) \tilde{v}_{\sigma / \tau}=\sum_{i} a_{i} \tilde{v}\left(A_{i}\right) \quad \in \mathbb{R}^{\left(\begin{array}{l}
n \\
2
\end{array}\right)} .
$$

The vectors $\tilde{v}\left(A_{i}\right)$ lie in the lineality space for $\left|A_{i}\right|=1$ and in $V_{\tau}$ otherwise, so taking the quotient by these spaces this sum reduces to zero. The claim of the lemma thus follows with the second part of Remark 2.11 .

Proposition 2.13 (Codimension- $k$ skeleton of $\mathcal{M}_{0, n}$ ). Let $k \in\{0, \ldots, n-3\}$. Then the codimension- $k$ skeleton $\mathcal{M}_{0, n}^{(n-3-k)}$ sk of $\mathcal{M}_{0, n}$ with the weight function $w: \mathcal{M}_{0, n}^{(n-3-k)} \rightarrow\{1\}$ is balanced. Hence, it is a tropical fan.

Proof. By Lemma 2.12 we can reduce the proof to the local situation of a star curve with a vertex of some valence $r \in\{4, \ldots, k+3\}$, i.e. to the balancing condition around the vertex $\tau_{\star}$ in $\mathcal{M}_{0, r}$.

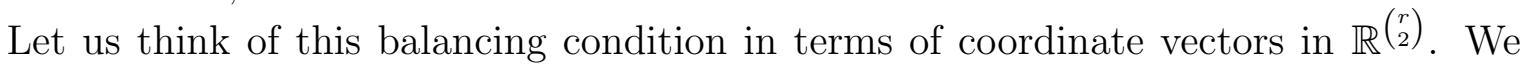
have to compute the sum $\tilde{v}$ of all normal vectors $\tilde{v}_{\sigma_{\star} / \tau_{\star}}$ arising from resolving the vertex of the star. Consider the first entry of this vector: here, we sum up 1 a number of times, a 1 for each type where the marked edges 1 and 2 lie on opposite sides of the bounded edge. By symmetry (i.e. no marked edge is distinguished), this sum is the same in each other entry of $\tilde{v}$. So $\tilde{v}$ is a multiple of the vector $(1, \ldots, 1)^{\top}$. Consider now the lineality space: summing up all vectors $d_{1}, \ldots, d_{r}$ gives $2 \cdot(1, \ldots, 1)^{\top}$. So $\tilde{v}$ is the zero vector modulo the lineality space. 
Proposition 2.14 (Codimension- $k$ skeleton of a Psi-class). Let $k \in\{0, \ldots, n-4\}$ and $i \in\{1, \ldots, n\}$. Then the codimension-k skeleton $\psi_{i}^{(n-4-k)}$ sk of the $i$-th Psi-class $\psi_{i}$ of $\mathcal{M}_{0, n}$ is balanced for the weight function $w: \psi_{i}^{(n-4-k)} \rightarrow\{1\}$. Hence, it is a tropical fan.

Proof. Again we can use Lemma 2.12 to reduce the proof to the local situation of a star with a vertex of some valence $r \in\{4, \ldots, k+4\}$. If the $i$-th unbounded edge is not adjacent to the chosen vertex then the computation is exactly the same as in the proof of Proposition 2.13, so let us assume that it is adjacent to the chosen vertex. Then, as in the picture of Example 2.15 below, we only have to consider resolutions of the star in which the $i$-th edge remains adjacent to a vertex of valence at least 4 .

Think again in terms of coordinate vectors in $\mathcal{M}_{0, r}$. Again, denote the sum of all normal vectors of these resolutions by $\tilde{v}$. Then the $(j, k)$-coordinates of $\tilde{v}$ with $j, k \neq i$ are all the same by symmetry, and likewise for the $(j, k)$-coordinates where $j=i$ or $k=i$. So if we set w.l.o.g. $i=1$ then we can write $\tilde{v}$ as

$$
\tilde{v}=(\underbrace{M, \ldots, M}_{r-1 \text { times }}, N, \ldots, N)^{\top}=M d_{1}+\frac{N}{2}\left(-d_{1}+d_{2}+\cdots+d_{r}\right)
$$

for suitable $M, N \in \mathbb{N}$. As this is a vector in the lineality space, the balancing condition follows.

Example 2.15 (Codimension-2 skeleton of $\psi_{1}$ in $\mathcal{M}_{0,7}$ ). The codimension-2 skeleton of $\psi_{1}$ in $\mathcal{M}_{0,7}$ is 1-dimensional, and thus there is only one balancing condition to check, namely that around the 0 -dimensional cell corresponding to the star curve with only one vertex. Allowed resolutions of this type are:

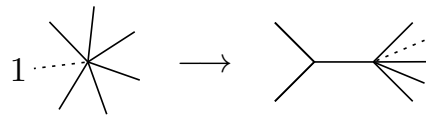

(a)

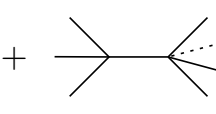

(b)

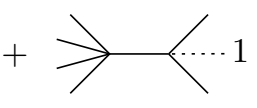

(c)

As in the proof of Proposition 2.14 let $\tilde{v}$ be the sum of all normal vectors of these resolutions. Write $\tilde{v}$ as $\tilde{v}=\tilde{v}_{(\mathrm{a})}+\tilde{v}_{(\mathrm{b})}+\tilde{v}_{(\mathrm{c})}$, where the summands denote the parts of $\tilde{v}$ arising from resolutions of type (a), (b), (c) as in the picture above, respectively. Note that each such type corresponds to various resolutions corresponding to the choice of labeling of the marked ends. In the array below, for each resolution type the coordinates of $\tilde{v}$ are listed, where $j, k \neq 1$.

\begin{tabular}{|c||c|c|c|c|}
\hline coordinate & $\tilde{v}_{(\mathrm{a})}$ & $\tilde{v}_{(\mathrm{b})}$ & $\tilde{v}_{(\mathrm{c})}$ & $\tilde{v}$ \\
\hline \hline$(1, j)$ & $\left(\begin{array}{l}5 \\
4\end{array}\right)=5$ & $\left(\begin{array}{l}5 \\
3\end{array}\right)=10$ & $\left(\begin{array}{l}5 \\
2\end{array}\right)=10$ & 25 \\
\hline$(j, k)$ & $2 \cdot\left(\begin{array}{l}4 \\
3\end{array}\right)=8$ & $2 \cdot\left(\begin{array}{l}4 \\
2\end{array}\right)=12$ & $2 \cdot\left(\begin{array}{l}4 \\
1\end{array}\right)=8$ & 28 \\
\hline
\end{tabular}

For example, the $(1, j)$-coordinate 10 of $\tilde{v}_{(\mathrm{b})}$ corresponds to the 10 choices of distributing the remaining labels on the ends in type (b) if the unbounded edge $j$ has been put at the left vertex. 
So we have

$$
\tilde{v}_{(\mathrm{a})}=4 \sum_{j=1}^{7} d_{j}-3 d_{1}, \quad \tilde{v}_{(\mathrm{b})}=6 \sum_{j=1}^{7} d_{j}-2 d_{1}, \quad \tilde{v}_{(\mathrm{c})}=4 \sum_{j=1}^{7} d_{j}+2 d_{1} .
$$

Note that it is not just the sum $\tilde{v}$ that is zero modulo the lineality space, but also the individual vectors $\tilde{v}_{(\mathrm{a})}, \tilde{v}_{(\mathrm{b})}, \tilde{v}_{(\mathrm{c})}$ corresponding to the resolution types themselves. In fact, the proof of Proposition 2.14 shows that the analogous statement holds for the (onedimensional) codimension- $(n-5)$ skeleton of a Psi-class in $\mathcal{M}_{0, n}$ for all $n \geqslant 5$ since the symmetry argument given there also applies if we only consider a single resolution type. ○

Remark 2.16 (Choice of weight function). Of course, the computations above depend on the chosen weight function. The moduli spaces $\mathcal{M}_{0, n}$ and the Psi-classes $\psi_{i}$, considered as tropical cycles, have all weights of their facets equal to 1, and thus it was natural in Propositions 2.13 and 2.14 to also equip the $k$-skeletons of these cycles with the constant weight function 1 . For other cycles such as for instance intersection products $\psi_{1}^{m_{1}} \cdot \ldots \cdot \psi_{n}^{m_{n}}$ of Psi-classes (where $m_{1}, \ldots, m_{n} \in \mathbb{N}_{0}$ ) this is in general no longer the case, especially when they are of codimension 2 or higher.

Now we want to explore the question of uniqueness of the weight function for certain tropical subfans of $\mathcal{M}_{0, n}$. This question is strongly related to the one of irreducibility introduced in [GKM]. To simplify notations, we will assume from now on that all weights of the cones in tropical cycles are non-zero.

Definition 2.17 (Irreducible tropical cycles). A tropical cycle $X$ in $V=\Lambda \otimes_{\mathbb{Z}} \mathbb{R}$ is said to be (globally) irreducible if there does not exist a tropical cycle $Y$ of the same dimension in $V$ such that $|Y| \subsetneq|X|$. Of course, this definition then applies to tropical fans as well.

Remark 2.18. Lemma 2.20 implies that, just as in the classical situation, the support of a tropical cycle can always be written as the union of the supports of irreducible tropical cycles. However, such a decomposition is in general not unique [GKM, Remark 2.19].

Remark 2.19. In Definition 2.17 the cones of $Y$ are just required to be contained in the union $|X|$ of the cones of $X$. However, the definition does not change if one requires all cones of $Y$ to actually be cones of $X$, just with possibly different weights. To see this, assume there is a tropical cycle $Y$ satisfying $|Y| \subsetneq|X|$. By passing to a common refinement with $X$, we can then first of all make sure that every cone of $Y$ is contained in a cone of $X$. But then all cones of $Y$ contained in the same cone of $X$ must have the same weight due to the balancing condition, and hence can be made into a single cone.

Lemma 2.20. A tropical cycle $X$ is irreducible if and only if "its weight function is unique up to a global multiple", i.e. if and only if for every cycle $Y$ of the same dimension and consisting of at most the cones of $X$ there is a rational number $\lambda \in \mathbb{Q}$ such that $w_{Y}(\sigma)=\lambda w_{X}(\sigma)$ for every cone $\sigma$ of $X$. 
Proof. " $\Rightarrow$ ": This is [GKM, Lemma 2.21].

" $\Leftarrow$ ": Let $Y$ be a cycle with $|Y| \subsetneq|X|$. By Remark 2.19 we can assume that each cone of $Y$ is a cone of $X$, so there must be a cone $\sigma$ of $X$ with $w_{Y}(\sigma)=0$. But this requires $\lambda=0$ in our assumption, so $Y$ would have to be the zero cycle.

Using this lemma the problem of checking irreducibility can often be reduced to a local computation. For this we need the concept of a cycle that is connected in codimension 1.

Definition 2.21 (Locally irreducible cycles, connected in codimension 1). Let $X$ be a tropical $k$-cycle.

1. The cycle $X$ is called locally irreducible if for each $(k-1)$-dimensional cone $\tau$ there is up to a common multiple only one choice of weights $w(\sigma)$ for the cones $\sigma$ adjacent to $\tau$ such that the balancing condition $\sum_{\sigma>\tau} w(\sigma) v_{\sigma / \tau}=0 \bmod V_{\tau}$ holds at $\tau$, see [R, Section 1.6].

2. The cycle $X$ is called connected in codimension 1 if any two $k$-dimensional cones $\sigma, \sigma^{\prime}$ in $X$ can be connected by a sequence $\sigma=\sigma_{0}, \ldots, \sigma_{m}=\sigma^{\prime}$ of $k$-dimensional cones in $X$ such that $\sigma_{i-1} \cap \sigma_{i}$ is of dimension $k-1$ for all $i=1, \ldots, m$ [BJSST, Section 3].

Remark 2.22. A criterion similar to Definition 2.17 holds also for locally irreducible cycles: we just have to apply Lemma 2.20 to the stars corresponding to each $\tau$ to see that a $k$-dimensional cycle is locally irreducible if and only if its stars at all $(k-1)$-dimensional cones are irreducible in the sense of Definition 2.17.

Lemma 2.23 (Criterion for irreducibility of a tropical cycle). Every locally irreducible tropical cycle that is connected in codimension 1 is also globally irreducible.

Proof. Let $\sigma, \sigma^{\prime} \in X^{(k)}$ be two top-dimensional cones in a $k$-dimensional cycle $X$. As $X$ is connected in codimension 1 there exist cones $\sigma_{i} \in X^{(k)}$ for $i \in\{1, \ldots, m\}$ forming a sequence $\sigma=\sigma_{0}, \ldots, \sigma_{m}=\sigma^{\prime}$ such that each intersection $\sigma_{i-1} \cap \sigma_{i}$ is a cone of dimension $k-1$. As $X$ is locally irreducible, the weight $w\left(\sigma_{i}\right)$ determines the weight $w\left(\sigma_{i-1}\right)$ uniquely. Hence, running through the sequence, we can conclude that $w(\sigma)$ determines $w\left(\sigma^{\prime}\right)$ uniquely and thus that $X$ is globally irreducible by Lemma 2.20 .

The converse of this lemma does not hold, see Example 2.27.

Lemma 2.24. $\mathcal{M}_{0, n}$ is connected in codimension 1 for each $n>2$.

Proof. Let $\sigma$ be a top-dimensional cone of $\mathcal{M}_{0, n}$, corresponding by Remark 2.3 to a combinatorial type of 3 -valent tropical curves.

First we show that $\sigma$ can be connected in codimension 1 (as in Definition 2.21 2) to a top-dimensional cone corresponding to a caterpillar tree, i.e. to a type of 3-valent curves for which the number $m$ of 3 -valent vertices that are not adjacent to any end is zero. So assume that $m>0$. As in the picture below we can then pick such a vertex $V$ that is not adjacent to any end but adjacent to at least one further vertex $W$ with two ends attached to it. 


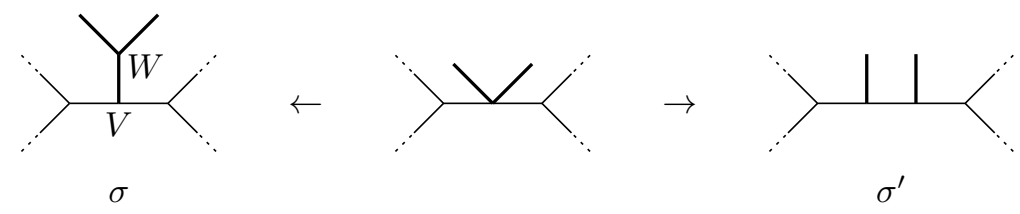

The picture then shows how $\sigma$ is connected in codimension 1 to another type $\sigma^{\prime}$ for which the value of $m$ is reduced by 1 - by contracting the edge joining $V$ and $W$ to a 4valent vertex, and then resolving this 4 -valent vertex again in a different way. Continuing this process, we can connect $\sigma$ in codimension 1 to a caterpillar tree.

But now any two caterpillar trees are connected in codimension 1 by a sequence of exchanges of neighboring ends as in the following picture:

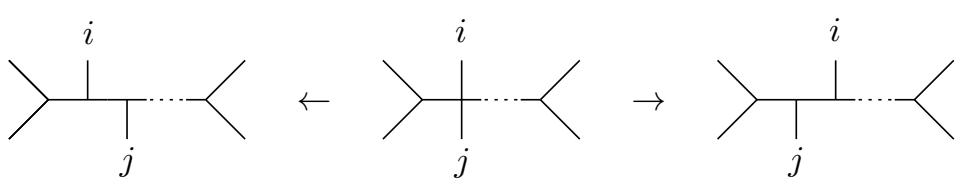

Hence we conclude that any two top-dimensional cones of $\mathcal{M}_{0, n}$ are connected in codimension 1 .

Remark 2.25. The statement of Lemma 2.24 is not new. It is noticed in [SS, Section 3 ] that the Plücker ideal $I_{2, n}$ is prime and that $\mathcal{G}(2, n)$ is the tropical variety defined by $I_{2, n}$. By [BJSST, Theorem 3.1] it follows that $\mathcal{G}(2, n)$ is connected in codimension one. When taking the quotient by its lineality space in order to obtain $\mathcal{M}_{0, n}$ this property is conserved.

We are now going to prove that for all $n>2$ the tropical moduli space $\mathcal{M}_{0, n}$ and its vital divisors, respectively Psi-classes are irreducible. These results also hold in classical algebraic geometry except for Psi-classes which are not divisors but only divisor classes: for $\bar{M}_{0, n}$ one can use the quotient description of [K2, Theorem 6.1], and the classical vital divisor $D^{S}$ is isomorphic to the product $\bar{M}_{0,|S|+1} \times \bar{M}_{0,\left|S^{c}\right|+1}$ of irreducible varieties [K1, Corollary 3.9], and hence is irreducible itself.

Proposition 2.26. $\mathcal{M}_{0, n}$ is irreducible for each $n>2$.

Proof. By Lemmata 2.23 and 2.24 it suffices to check local irreducibility. We have to look at the weights of cones corresponding to the resolving curves of a curve with one 4-valent vertex. By Lemma 2.12 we can assume that this curve is just a star with one 4-valent vertex in the middle, living in $\mathcal{M}_{0,4}$.

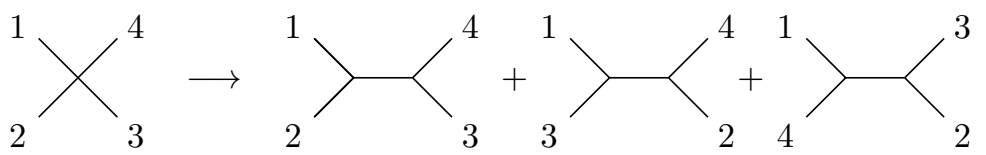


So the task translates to finding non-trivial solutions $\left(a_{1}, \ldots, a_{7}\right)$ for the linear system of equations

$$
a_{1} \tilde{v}(\{1,2\})+a_{2} \tilde{v}(\{1,3\})+a_{3} \tilde{v}(\{1,4\})=a_{4} d_{1}+a_{5} d_{2}+a_{6} d_{3}+a_{7} d_{4}
$$

in $\mathbb{R}^{\left(\begin{array}{l}4 \\ 2\end{array}\right)}=\mathbb{R}^{6}$. Solving this system gives $a_{i}=1$ for all $i \in\{1, \ldots, 7\}$ up to a multiple. Hence $\mathcal{M}_{0, n}$ is locally irreducible and thus also globally irreducible.

Next, we want to study the irreducibility of codimension-1 cycles in $\mathcal{M}_{0, n}$ - in particular of Psi-classes and vital divisors. In this case we can often not use Lemma 2.23 as the cycles in question are not locally irreducible. Let us start by illustrating this fact with an example that will also be needed later.

Example 2.27 (Difference between local and global irreducibility). The following picture shows a type of curves corresponding to a codimension- 2 cell $\tau$ in $\mathcal{M}_{0,6}$ with the types of its six adjacent codimension-1 cells $\sigma_{1}, \ldots, \sigma_{6}$ :
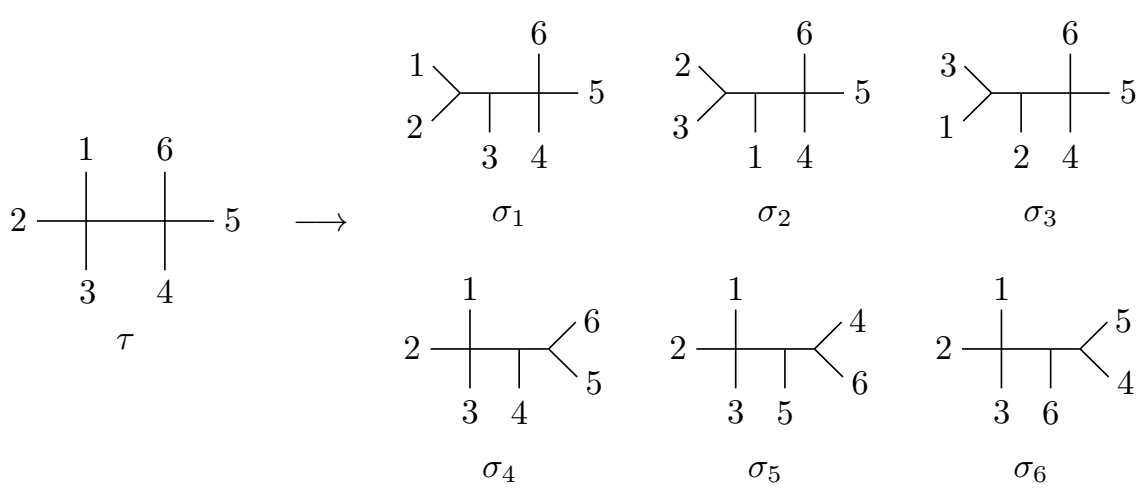

By Lemma 2.12 and the balancing condition in $\mathcal{M}_{0,4}$ we have $v_{\sigma_{1} / \tau}+v_{\sigma_{2} / \tau}+v_{\sigma_{3} / \tau}=0$ and $v_{\sigma_{4} / \tau}+v_{\sigma_{5} / \tau}+v_{\sigma_{6} / \tau}=0$. In fact, an easy computation in coordinates shows that the linear equation $\sum_{i=1}^{6} a_{i} v_{\sigma_{i} / \tau}=0$ is satisfied in $\mathcal{M}_{0,6}$ if and only if $a_{1}=a_{2}=a_{3}$ and $a_{4}=a_{5}=a_{6}$. All six resolutions above contribute to the vital divisor $D^{\{1,2,3\}}$ with the same non-zero weight -1 by Proposition 2.9. This means that we can scale $a_{1}, a_{2}, a_{3}$ by one multiple and $a_{4}, a_{5}, a_{6}$ independently by another one in order to satisfy the equation $\sum_{i=1}^{6} a_{i} v_{\sigma_{i} / \tau}=0$. Therefore $D^{\{1,2,3\}}$ is not locally irreducible at $\tau$. We will see in Proposition 2.34 however that vital divisors are always globally irreducible.

Lemma 2.28. Let $Y$ be a codimension-1 cycle in $\mathcal{M}_{0, n}$. Let $\sigma$ be a top-dimensional cone of $Y$; by Remark 2.3 its corresponding curves have exactly one 4-valent vertex. Denote by $A, B, C, D \subset\{1, \ldots, n\}$ the sets of marked ends behind each of the four edges adjacent to this vertex (in any order). Then the weight $w(\sigma)$ in $Y$ depends only on the sets $A, B, C, D$ (and not on the subtrees behind the 4-valent vertex).

Proof. Let $\tau^{\prime}$ be a codimension-1 cell of $Y$, i.e. a codimension-2 cell in $\mathcal{M}_{0, n}$. By Remark 2.3 the corresponding curves have either two 4 -valent vertices or one 5 -valent vertex, with all other vertices being 3-valent. The case of a 5 -valent vertex is irrelevant for the claim of 
the lemma and will be treated in Proposition 2.30. Let us consider the case when $\tau^{\prime}$ has two 4-valent vertices. Without loss of generality we may then assume that the ends are labeled in such a way that there is a forgetful map $\mathcal{M}_{0, n} \rightarrow \mathcal{M}_{0,6}$ sending $\tau^{\prime}$ to the cone $\tau$ in Example 2.27 above. By Remark 2.11 the top-dimensional cones $\sigma_{1}^{\prime}, \ldots, \sigma_{6}^{\prime}$ in $Y$ adjacent to $\tau^{\prime}$ are in one-to-one correspondence with the cones $\sigma_{1}, \ldots, \sigma_{6}$ in $\mathcal{M}_{0,6}$ of Example 2.27. Now let $a_{i}$ be the weight of $\sigma_{i}^{\prime}$ in $Y$ for $i=1, \ldots, 6$. By the balancing condition we have $\sum_{i} a_{i} v_{\sigma_{i}^{\prime} / \tau_{i}}=0$ in $\mathcal{M}_{0, n}$. Applying the (linear) forgetful map $\mathcal{M}_{0, n} \rightarrow \mathcal{M}_{0,6}$ we conclude that $\sum_{i} a_{i} v_{\sigma_{i} / \tau_{i}}=0$ in $\mathcal{M}_{0,6}$ as well, which by Example 2.27 means that $a_{1}=a_{2}=a_{3}$ and $a_{4}=a_{5}=a_{6}$. In particular, the types $\sigma_{1}^{\prime}, \sigma_{2}^{\prime}, \sigma_{3}^{\prime}$ have the same weight in $Y$. Applying this to the labeling where the forgetful map sends the marked ends in $B, C, D$ to $x_{4}$, $x_{5}, x_{6}$, respectively, we see by the argument of the proof of Lemma 2.24 that any two top-dimensional cells of $Y$ that just differ by the arrangement of the markings in $A$ behind one of the edges of the 4 -valent vertex must have the same weight. As the same holds for $B, C, D$ as well, the lemma follows.

Lemma 2.29. $\psi_{i}$ is connected in codimension 1 for each $i \in\{1, \ldots, n\}$.

Proof. Let $\sigma$ be a top-dimensional cone in $\psi_{i}$. As in the picture below on the left it corresponds to a combinatorial type of tropical curves with exactly one 4-valent vertex $V$ adjacent to the marked point $x_{i}$, all other vertices being 3 -valent.

By the proof of Lemma 2.28 we see that $\sigma$ can be connected in codimension 1 to any other such type where the trees behind the edges of $V$ have been replaced by caterpillars, and where within each such caterpillar the marked points have been ordered in a given way. The picture below then shows that it is also possible to connect such a cone in codimension 1 to the one where a marked point $x_{j}$ with $j \neq i$ changes its place from behind one of the edges of $V$ to another - through a codimension-1 cone in $\psi_{i}$ corresponding to curves with a 5 -valent vertex.

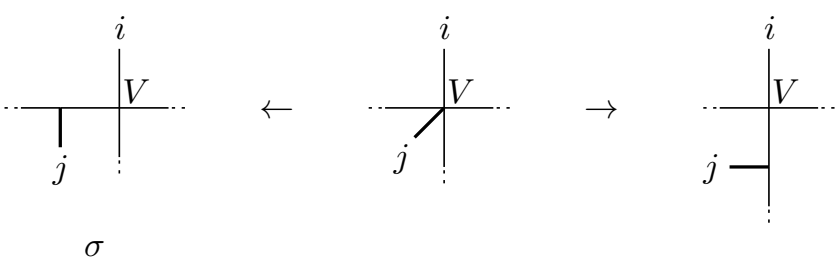

Using these steps we see that $\sigma$ can be connected in codimension 1 to any other top-dimensional cone of $\psi_{i}$.

Proposition 2.30. Each Psi-class $\psi_{i}$ in $\mathcal{M}_{0, n}$ is irreducible.

Proof. Assume w.l.o.g. $i=1$. As in the proof of Proposition 2.26 it suffices by Lemmata 2.23 and 2.29 to check local irreducibility at each codimension-1 cone of $\psi_{1}$. There are two types of these cones: those with two 4-valent vertices (one of them adjacent to $x_{1}$ ) and those with one 5 -valent vertex (adjacent to $x_{1}$ ). In the former case only the vertex that is not adjacent to $x_{1}$ can be resolved in $\psi_{1}$, and the computation to check local irreducibility is exactly the same as in Proposition 2.26. In the latter case we again apply 
Lemma 2.12 to reduce the situation to the resolutions of a star in $\mathcal{M}_{0,5}$ such that the marking $x_{1}$ remains adjacent to a 4 -valent vertex. There are six such resolving curves of the 5 -valent vertex, corresponding to a choice of two of the marked ends $x_{2}, \ldots, x_{5}$ to put at the 4 -valent vertex together with $x_{1}$ and the bounded edge. Hence, this time the linear system of equations reads

$$
\begin{aligned}
a_{1} \tilde{v}(\{2,3\}) & +a_{2} \tilde{v}(\{2,4\})+a_{3} \tilde{v}(\{2,5\})+a_{4} \tilde{v}(\{3,4\})+a_{5} \tilde{v}(\{3,5\})+a_{6} \tilde{v}(\{4,5\}) \\
= & \sum_{j=7}^{11} a_{j} d_{j-6}
\end{aligned}
$$

in $\mathbb{R}^{\left(\begin{array}{l}5 \\ 2\end{array}\right)}=\mathbb{R}^{10}$. Solving this system gives up to a multiple $a_{j}=1$ for $j=1, \ldots, 7$ and $a_{j}=2$ for $j=8, \ldots, 11$ as the only solution. Hence $\psi_{i}$ is locally irreducible and thus also globally irreducible.

Remark 2.31. Using similar arguments as in Lemmata 2.24 and 2.29 one can show that the codimension- $k$ skeletons of the moduli spaces $\mathcal{M}_{0, n}$ as well as of the Psi-classes $\psi_{i}$ (see Propositions 2.13 and 2.14) are connected in codimension 1 as well. However, they are in general not irreducible, as the following example shows.

Example 2.32 (Irreducibility of other tropical fans in $\mathcal{M}_{0, n}$ ). Consider the (one-dimensional) codimension- $(n-5)$ skeleton of the Psi-class $\psi_{1}$ in $\mathcal{M}_{0, n}$ for $n \geqslant 6$, corresponding to resolutions of the star curve with one $n$-valent vertex that keep $x_{1}$ at a vertex of valence at least 4. We have seen at the end of Example 2.15 that in this case it is not only the sum of all normal vectors of the possible resolutions that sum up to zero modulo the lineality space, but also the vectors of each individual resolution type, i.e. when fixing how many marked ends should be adjacent to $x_{1}$. In particular, as there is more than one such resolution type if $n \geqslant 6$, this means that there are several choices of weights satisfying the balancing condition and thus that the codimension- $(n-5)$ skeleton of $\psi_{1}$ in $\mathcal{M}_{0, n}$ is not locally irreducible. As for this cycle there is only one balancing condition to check it follows that it is not globally irreducible either.

A similar argument can be used to show that other codimension- $k$ skeletons of a Psi-class or of $\mathcal{M}_{0, n}$ also fail to be locally irreducible. In general this does not imply however that these cycles are not globally irreducible either (e.g. we will see in the proof of Proposition 2.34 that the vital divisors of $\mathcal{M}_{0, n}$ are globally but not locally irreducible). $\circ$

The following example will be of use in the remainder.

Example 2.33 (Irreducible divisors in $\mathcal{M}_{0,5}$ ). In $\mathcal{M}_{0,5}$ the problem of finding irreducible divisors is equivalent to determine all minimal linear dependent sets of the 10 vectors $v(I)$ where $I \subset\{1, \ldots, 5\}$ and $|I|=2$, or in other words to find all circuits of the vector matroid given by these vectors. An explicit computation shows that there are two kinds of irreducible divisors: those containing 4 cones in their support and those with 6 cones. Up to multiples, these divisors are the following: 
- 4 cones: The only effective divisors with four cones are the cycles $\psi_{i}^{\natural}$ for $i=1, \ldots, 5$ given as the difference of the 1-skeleton and $\psi_{i}$ - for example $\psi_{1}^{\natural}$ consists of the cones $v(\{1,2\}), v(\{1,3\}), v(\{1,4\})$, and $v(\{1,5\})$, each with weight 1 . The only other divisors with four cones are the ten vital divisors $D^{S}$ of $\mathcal{M}_{0,5}$. They have respectively three cones of weight 1 and one of weight -1 (see Proposition 2.9).

- 6 cones: The Psi-classes $\psi_{i}$ are the only effective irreducible divisors with six cones. There are ten more irreducible divisors with six cones, given by $\psi_{i}^{\natural}-\psi_{j}^{\natural}=: V^{\{i, j\}}$ for $i>j$.

Proposition 2.34. For $n>3$ all vital divisors $D^{S}$ in $\mathcal{M}_{0, n}$ are irreducible.

Proof. The case $n=4$ is clear as the only vital divisor corresponds to the star with a 4 -valent vertex. So let $n \geqslant 5$, and let $S \subset\{1, \ldots, n\}$ be a subset with $2 \leqslant|S| \leqslant n-2$, giving rise to a vital divisor $D^{S}$ in $\mathcal{M}_{0, n}$. By Proposition 2.9 the cones occurring in $D^{S}$ correspond to curves with one 4-valent vertex such that, if $A, B, C, D$ denote the sets of marked points behind the 4 -valent vertex, the given set $S$ can be written as a union of some of the sets $A, B, C, D$. We have to show that up to a global multiple there is only one weight distribution on these cones giving rise to a tropical cycle. By relabeling the subsets (and possibly replacing $S$ by $S^{c}$ ) we may assume w.l.o.g. that $S=A$ or $S=A \cup B$.

By Lemma 2.28 we know already that the weights of these cones can only depend on the sets $A, B, C, D$; we denote these weights by $w(A, B, C, D)$. So to prove irreducibility it suffices to show that

1. $w\left(A, B, C_{1}, D \cup C_{2}\right)=w(A, B, C, D)$ if $S=A \cup B, C=C_{1} \sqcup C_{2}$, and $C_{1}, C_{2} \neq \emptyset$,

2. $w\left(A \cup B, C_{1}, C_{2}, D\right)=-w(A, B, C, D)$ if $S=A \cup B, C=C_{1} \sqcup C_{2}$, and $C_{1}, C_{2} \neq \emptyset$,

since these two operations can transform any partition of $\{1, \ldots, n\}$ into four subsets occurring in $D^{S}$ into any other.

To prove this, consider a codimension-1 cone in $D^{S}$ having one 5 -valent vertex, with sets of marked points in $A, B, C_{1}, C_{2}, D$ behind its adjacent edges. Since $S=A \cup B$, only 4 of the 10 resolutions of this cone in the codimension-1 skeleton of $\mathcal{M}_{0, n}$ occur in $D^{S}$ :
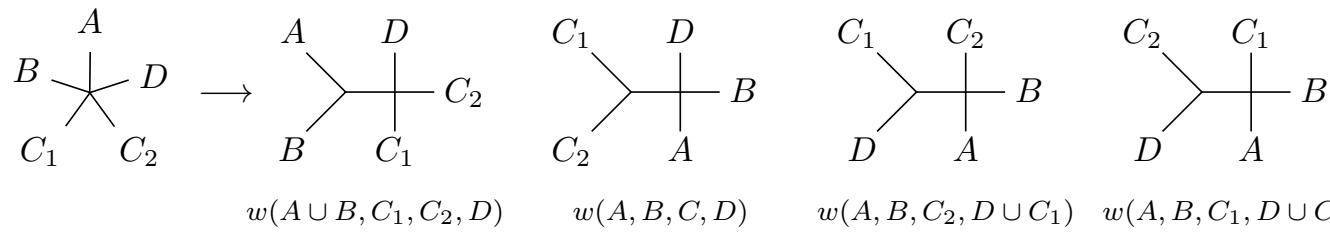

$w\left(A, B, C_{2}, D \cup C_{1}\right) \quad w\left(A, B, C_{1}, D \cup C_{2}\right)$

Now we map these cones to $\mathcal{M}_{0,5}$ by the forgetful map that sends the marked ends in $A, B, C_{1}, C_{2}, D$ to $x_{1}, x_{2}, x_{3}, x_{4}, x_{5}$, respectively. The 4 cones occurring above are then exactly mapped to the four cones in the vital divisor $D^{\{1,2\}}$ in $\mathcal{M}_{0,5}$ of Example 2.10. As this divisor is irreducible (see Example 2.33), we conclude that there is up to a global multiple only one choice of weights for the four cones above. By the explicit computation of Example 2.10 these weights satisfy $-w\left(A \cup B, C_{1}, C_{2}, D\right)=w\left(A, B, C_{1}, D \cup C_{2}\right)=$ $w(A, B, C, D)$, proving both (a) and (b). 
Remark 2.35. The proofs of Propositions 2.30 and 2.34 suggest that, once we know that a divisor in $\mathcal{M}_{0, n}$ is locally irreducible, we might be able to conclude by some "connectivity" argument that the divisor is also (globally) irreducible. Unfortunately, the proof of this connectivity is very complicated for increasing $n$ since the number of possible cones contained in the support of such a divisor increases exponentially. So far, we are just able to show the equivalence of local and global irreducibility of a divisor in the case $n=6$ (for $n=5$ this is trivial since there is only one balancing condition):

Proposition 2.36. In $\mathcal{M}_{0,6}$ every locally irreducible divisor is also globally irreducible.

Proof. Let $D$ be a locally irreducible divisor. We have to show that its weight function is unique up to a global multiple in the sense of Lemma 2.20.

To prove this, let us introduce the following notation. A codimension-2 cone $\tau$ of $D$ will be called relevant if it corresponds to curves with one 5-valent vertex (rather than to curves with two 4 -valent vertices). Note that $D$ is determined uniquely by the weights of all cells adjacent to relevant cones (hence the name) since every codimension-1 cell is adjacent to such a relevant codimension- 2 cone. Moreover, in $\mathcal{M}_{0,6}$ all relevant cones correspond to curves having two markings $i$ and $j$ on one side of the bounded edge and four on the other. We will use the notation $i j$ for this cone.

As $D$ is locally irreducible we know that the weight combination of the codimension1 cells around a relevant cone $\tau$ must be a multiple of the irreducible types of divisors in $\mathcal{M}_{0,5}$ listed in Example 2.33. If the weights around $\tau$ are not all 0 we will call the choice of type the resolution behavior at $\tau$, and the corresponding multiple the weight of $\tau$. However, in $\mathcal{M}_{0,6}$, where each curve has 6 markings, we have to be careful how we split up the six markings over the subtrees behind the five bounded edges adjacent to the 5-valent vertex. This means that all types of Example 2.33 correspond to several resolution behaviors. The following list shows all possible resolution behaviors at the cone 12 (up to permutation of the labels of the markings $3,4,5,6$ ). In this list, we understand by new bounded edge the bounded edge appearing when resolving the 5-valent vertex.

- $\psi_{3}$, where we require the marking 3 to be at the 4 -valent vertex in the resolving curves;

- $\psi_{12}$ (not really a Psi-class!) corresponding to resolving the 5-valent vertex where the subtree consisting of the markings 1 and 2 is required to stay at the 4 -valent vertex;

- $V^{\{5,6\}}$, where either the marking 5 or 6 should lie at the 4 -valent vertex (but not both);

- $V^{\{12,3\}}$, where the marking 3 or the subtree consisting of the markings 1 and 2 should be at the 4 -valent vertex, but not both;

- $\psi_{3}^{\natural}$, where the marking 3 is not allowed to lie at the 4 -valent vertex;

- $\psi_{12}^{\natural}$, where the subtree consisting of the markings 1 and 2 is not allowed to lie at the 4 -valent vertex; 
- $D^{\{5,6\}}$, where the markings 5 and 6 lie both on the side of the new bounded edge without 4-valent vertex, or both on the side of the new bounded edge with the 4 -valent vertex;

- $D^{\{12,3\}}$, where the marking 3 and the subtree consisting of the markings 1 and 2 lie both on the side of the new bounded edge without 4-valent vertex, or both on the side of the new bounded edge with the 4-valent vertex.

For some resolution behaviors, we show the corresponding adjacent codimension-1 cells occurring in $D$ with non-zero weight in the picture below, where unmarked ends are understood to be labeled with the remaining markings in any possible way.

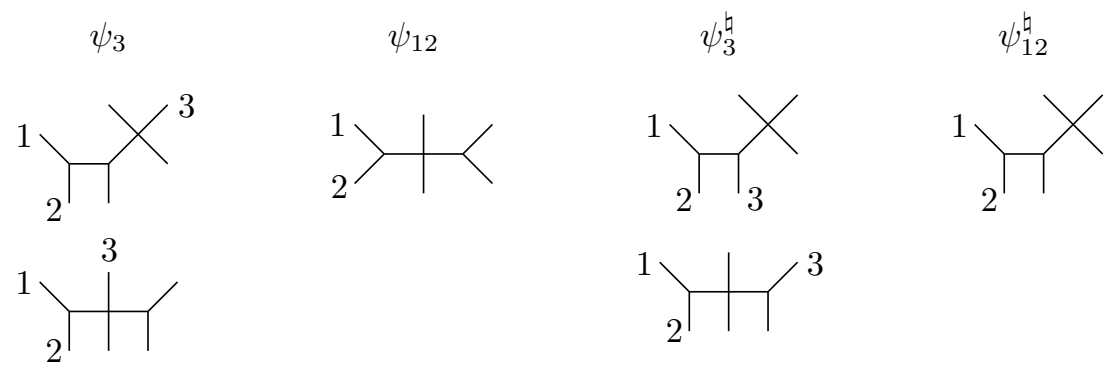

The main idea of the proof is now the following: pick a relevant codimension- 2 cone, say 12 , such that the weights of $D$ around it are not all 0 . If we prescribe the resolution behavior and the weight at 12 this will determine the weight (not necessarily the resolution behavior however) of all other relevant codimension- 2 cones adjacent to codimension-1 cells occurring in the chosen resolution behavior at 12. For example, if we choose the resolution behavior $\psi_{3}$ at 12 as above, the picture below shows the type 12 (on the left) and an adjacent codimension-1 cell with non-zero coefficient in $\psi_{3}$ in the middle. As this cell is also adjacent to the relevant codimension- 2 cone 56 (on the right), this means that the weight of 56 is fixed by that of 12 .

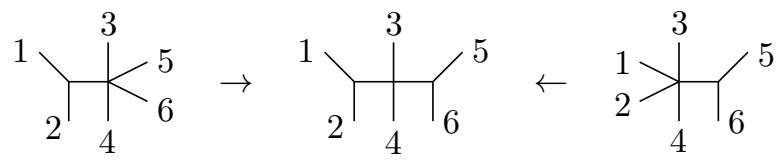

This argument can be extended slightly by using in addition the result of Lemma 2.28 that the weights of codimension-1 cells in divisors depend only on the distribution of the marked points behind the 4 -valent vertex. For example, the following picture shows that the weight of 14 is also fixed by the weight (and the resolution behavior) of 12 .

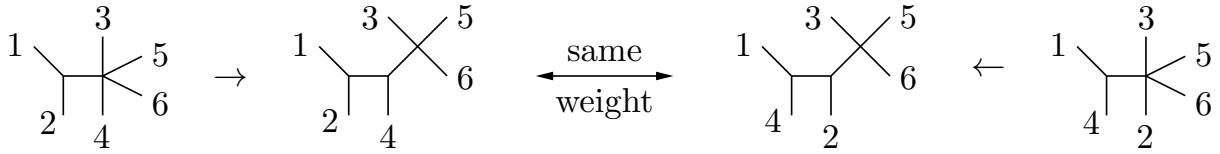


Using these arguments, the following list shows, when fixing a certain resolution behavior at 12 , the weights of which other relevant codimension- 2 cones are fixed by the weight of 12 . The column on the right shows the number of relevant codimension- 2 cones whose weight is fixed by that of 12 .

\begin{tabular}{|l|l|r|}
\hline$\psi_{3}$ & $12,14,15,16,24,25,26,45,46,56$ & 10 \\
$\psi_{12}$ & $12,34,35,36,45,46,56$ & 7 \\
$V^{\{5,6\}}$ & $12,15,16,25,26,35,36,45,46$ & 9 \\
$V^{\{12,3\}}$ & $12,14,15,16,24,25,26,34,35,36$ & 10 \\
$\psi_{3}^{\natural}$ & $12,13,23,34,35,36$ & 6 \\
$\psi_{12}^{\natural}$ & $12,13,14,15,16,23,24,25,26$ & 9 \\
$D^{\{5,6\}}$ & $12,13,14,23,24,34,56$ & 7 \\
$D^{\{12,3\}}$ & $12,13,23,45,46,56$ & 6 \\
\hline
\end{tabular}

Note that there are $\left(\begin{array}{l}6 \\ 2\end{array}\right)=15$ relevant codimension- 2 types in total. So if we assume that $D$ is reducible, this means that a resolution behavior of $\psi_{3}$ or $V^{12,3}$ as in the table above is impossible since this fixes 10 weights already, leaving only 5 of the weights of relevant cones undetermined - which is not enough for another independent weight since each weight has to occur in at least 6 relevant cones by the table above.

To rule out the other resolution behaviors we cannot use a pure counting argument alone, but rather look in detail at the precise cones whose weights are determined by a fixed weight in a certain resolution behavior. A straightforward but tedious case-by-case analysis using the table above shows that it is not possible to fix the resolution behaviors and independent weights at two relevant cones without contradictions, i.e. such that the sets of relevant cones whose weights are determined by the two initial ones are disjoint. This shows that $D$ must in fact be irreducible.

Since the techniques used in Proposition 2.36 are limited to $n=6$, the following question remains open:

Question 2.37. Let $n>6$. Is any locally irreducible divisor in $\mathcal{M}_{0, n}$ also globally irreducible?

\section{The set of points in special position for rational $n$-marked plane tropical curves}

One natural extension of the notion of rational $n$-marked abstract tropical curve is the rational $n$-marked parametrized tropical curve introduced below, which we consider to be the tropical analogue of a rational $n$-marked stable map in classical algebraic geometry. Recall that for a given smooth complex projective variety $X, n \in \mathbb{N}$ and a homology class $\beta \in H_{2}(X, \mathbb{Z})$ a rational $n$-marked stable map (into $X$ ) is a collection $\left(C ; x_{1}, \ldots, x_{n}, f\right)$ of a complete connected nodal curve $C$ of arithmetic genus 0 , smooth points $x_{i} \in C$ which are pairwise disjoint, and a morphism $f: C \rightarrow X$ satisfying $f([C])=\beta$ s.t. every 
irreducible component that is mapped to a point in $X$ contains at least 3 special points, i.e. markings or intersection points with other components (stability). Two stable $n$ marked maps $\left(C ; x_{1}, \ldots, x_{n}, f\right)$ and $\left(\tilde{C} ; \tilde{x}_{1}, \ldots, \tilde{x}_{n}, \tilde{f}\right)$ are isomorphic if there exists an isomorphism $\varphi: C \rightarrow \tilde{C}$ s.t. $\varphi\left(x_{i}\right)=\tilde{x}_{i}$ and $\tilde{f} \circ \varphi=f$. The space $\bar{M}_{0, n}(X, \beta)$ is the set of isomorphism classes of rational $n$-marked stable maps; it carries the structure of a smooth projective variety $[\mathrm{B}, \mathrm{FP}]$.

In both the tropical and the algebraic setup one can then count such curves through given points, where the number of these points has been chosen so that one expects a finite answer. We are interested in the description of the set of points in special position for these counting problems. Remember that in the classical situation, when the ambient space for the rational stable maps is $X=\mathbb{P}^{2}$, it is known that the points in special position - in the sense that there are infinitely many curves passing through them - form a subvariety of $\left(\mathbb{P}^{2}\right)^{n}$ (see [EGAIV, Corollaire 13.1.5] applied to the (product) evaluation map). In the tropical context a more striking result holds, namely that these points form a tropical subfan of $\left(\mathbb{R}^{2}\right)^{n}$ of codimension one, which we can describe as a push-forward of some divisor in $\mathcal{M}_{0, n}$. To be more precise, we deal here with two notions of "points in special position" which both as sets arise as a divisor pushed forward by the evaluation map. We restrict ourselves to the case where the ambient space is $\mathbb{R}^{2}$ as our arguments just hold there.

But let us start by recalling the necessary tropical vocabulary. More details about the following can be found in [GKM, section 4].

Definition 3.1 (Rational $n$-marked (labeled) parametrized tropical curve of degree $\Delta$ in $\mathbb{R}^{2}$. We denote a tuple $\left(v_{1}, \ldots, v_{m}\right) \in\left(\mathbb{Z}^{2} \backslash\{0\}\right)^{m}$ by $\Delta$.

A rational $n$-marked (labeled) parametrized tropical curve of degree $\Delta$ in $\mathbb{R}^{2}$ is a pair $(C, h)$ consisting of a rational $(n+m)$-marked abstract tropical curve $C=\left(\Gamma ; x_{1}, \ldots, x_{n+m}\right)$ and a continuous map $h: \Gamma \rightarrow \mathbb{R}^{2}$ such that the following holds.

- The map $h$ is integer affine linear on each edge $E$ of $\Gamma$, i.e. of the form $h(t)=a+v t$ for some $a \in \mathbb{R}^{2}$ and $v \in \mathbb{Z}^{2}$. If we start parametrizing $E$ at the vertex $V \in \partial E$ we call $v$ the direction $v(E, V)$ of $E$ with respect to $V$.

- At each vertex $V$ the balancing condition

$$
\sum_{E: V \in \partial E} v(E, V)=0
$$

holds.

- The direction of $x_{i}$ is 0 for all $i=1, \ldots, n$ (contracted ends).

- The direction of $x_{i}$ is $v_{i-n}$ for all $i=n+1, \ldots, n+m$ (non-contracted ends).

Let $V$ be a 3 -valent vertex which is not adjacent to a contracted end. Let $w_{1}, w_{2}, w_{3}$ be the direction vectors of its adjacent edges. Then we define the multiplicity mult $(V)$ of $V$ to be $\left|\operatorname{det}\left(w_{1} \mid w_{2}\right)\right|=\left|\operatorname{det}\left(w_{1} \mid w_{3}\right)\right|=\left|\operatorname{det}\left(w_{2} \mid w_{3}\right)\right|$. 
In our pictures we will usually only draw the image curve $h(\Gamma)$ together with the points $h\left(x_{1}\right), \ldots, h\left(x_{n}\right)$. This image then has $m$ (labeled) unbounded edges whose directions are contained in $\Delta$. We can think of the degree $\Delta$ as the tropical equivalent of the homology class of an algebraic stable map. Note that the stability condition translates to the requirement that $\Gamma$ has at least 3 -valent vertices.

In the following we will sometimes abbreviate the term "rational $n$-marked (labeled) parametrized tropical curve of degree $\Delta$ in $\mathbb{R}^{2}$ " by "parametrized tropical curve".

Construction 3.2. Two parametrized tropical curves $(C, h)$ and $(\tilde{C}, \tilde{h})$ are isomorphic if there is an isomorphism $\varphi$ between the underlying abstract tropical curves $C$ and $\tilde{C}$ satisfying $\tilde{h} \circ \varphi=h$. The parameter space of rational $n$-marked (labeled) parametrized tropical curves of given degree $\Delta$ in $\mathbb{R}^{2}$ modulo isomorphisms is denoted by $\mathcal{M}_{0, n}^{\text {lab }}\left(\mathbb{R}^{2}, \Delta\right)$. Observe that the directions of the bounded edges in a parametrized tropical curve are not fixed by $\Delta$. But when fixing a combinatorial type (see Remark 2.3) in $\mathcal{M}_{0, n+m}$ for $m=|\Delta|$ there is a unique choice for the directions of the bounded edges for a parametrized tropical curve of degree $\Delta$ in $\mathbb{R}^{2}$ such that the underlying graph $\Gamma$ is of this combinatorial type. Hence there is a bijection between combinatorial types of the moduli spaces $\mathcal{M}_{0, n}^{\text {lab }}\left(\mathbb{R}^{2}, \Delta\right)$ and $\mathcal{M}_{0, n+m}[\mathrm{GKM}$, Lemma 4.6]. Note that the number of combinatorial types in $\mathcal{M}_{0, n}^{\text {lab }}\left(\mathbb{R}^{2}, \Delta\right)$ is finite too for this reason.

Coordinates in $\mathcal{M}_{0, n}^{\text {lab }}\left(\mathbb{R}^{2}, \Delta\right)$ are given by the position of a root vertex in $\mathbb{R}^{2}$ of the parametrized tropical curve and the lengths of the bounded edges (see [GM2, Proposition 2.11]). Hence, given a parametrized tropical curve in $\mathcal{M}_{0, n}^{\text {lab }}\left(\mathbb{R}^{2}, \Delta\right)$ we can send it to the image under the map $v$ of its underlying graph $\Gamma$ (see Construction 2.2). So we get an isomorphism of polyhedral complexes [GKM, Proposition 4.7]

$$
\mathcal{M}_{0, n}^{\mathrm{lab}}\left(\mathbb{R}^{2}, \Delta\right) \stackrel{\cong}{\rightarrow} v\left(\mathcal{M}_{0, n+m}\right) \times \mathbb{R}^{2} .
$$

In particular, we can consider $\mathcal{M}_{0, n}^{\text {lab }}\left(\mathbb{R}^{2}, \Delta\right)$ as a tropical fan of dimension $(n+m-3)+2$.

Definition 3.3 (More intersection theory and tropical morphisms). We extend the definitions of Definition 2.4.

(a) Let $X$ and $Y$ be two $k$-cycles. After possibly adequately refining $X$ and $Y$ we can construct a $k$-cycle on $X \cup Y$, called the sum of the cycles $X$ and $Y$, which is denoted by $X+Y$ [AR, Construction 2.13].

(b) A morphism $f: X \rightarrow Y$ of cycles $X$ and $Y$ (resp. of fans $X$ and $Y$ ) is a $\mathbb{Z}$-linear map, i.e. a map $\tilde{f}:|X| \rightarrow|Y|$ induced by a $\mathbb{Z}$-linear map between the underlying lattices $\Lambda_{X}$ and $\Lambda_{Y}$.

(c) Let $f: X \rightarrow Y$ be a morphism of a cycle $X$ to an $m$-cycle $Y, Z$ an $n$-cycle in $X$ where $n \leqslant m$. Using an appropriate refinement of $X$ we can assume that the image of each cone in $X$ is a cone of $Y$. We define the push-forward of $Z$ along $f$ by $f_{*}(Z)=\{f(\sigma) \mid \sigma \in Z\}$. This polyhedral complex $f_{*}(Z)$ becomes an $n$-cycle in 
$Y$ by giving the weights

$$
w_{f_{*}(Z)}\left(\sigma^{\prime}\right)=\sum_{\substack{\sigma \in Z \\ f(\sigma)=\sigma^{\prime}}} w_{Z}(\sigma) \cdot\left|\Lambda_{\sigma^{\prime}} / f\left(\Lambda_{\sigma}\right)\right|
$$

to the $n$-dimensional cones $\sigma^{\prime} \in Y$ in the image of $Z$ under $f$ [AR, Proposition 4.6], where $\Lambda_{\sigma}$ is the sublattice of $\Lambda_{X}$ generated by $\sigma$.

(d) The tropical Psi-classes of Definition 2.5 can be written as the divisors associated to certain rational functions [KM, Chapter 3]. As such, we can intersect several Psi-classes by consecutively intersecting with these rational functions. The product $\psi_{1}^{k_{1}} \cdot \ldots \cdot \psi_{n}^{k_{n}}$ is then a cycle whose support consists of all curves such that a vertex with the markings $i_{1}, \ldots, i_{m}$ has valence at least $k_{i_{1}}+\cdots+k_{i_{m}}+3[\mathrm{KM}$, Chapter $4]$.

Definition 3.4 (Evaluation maps). For all $i=1, \ldots, n$ define the $i$-th evaluation map by

$$
\mathrm{ev}_{i}: \mathcal{M}_{0, n}^{\mathrm{lab}}\left(\mathbb{R}^{2}, \Delta\right) \rightarrow \mathbb{R}^{2},(C, h) \mapsto h\left(x_{i}\right)
$$

These maps are well-defined and are morphisms of fans [GKM, Proposition 4.8] using Construction 3.2. The evaluation map

$$
\mathrm{ev}:=\prod_{i=1}^{n} \mathrm{ev}_{i}: \mathcal{M}_{0, n}^{\mathrm{lab}}\left(\mathbb{R}^{2}, \Delta\right) \rightarrow \mathbb{R}^{2 n}
$$

is a morphism of fans, too.

Definition 3.5 (Two versions of points in special position). Fix $n>0$ and a degree $\Delta$ such that $n=|\Delta|-1$ (i.e. so that the source and target of ev have the same dimension and we expect a finite number of curves of degree $\Delta$ through $n$ given points). A collection $Q=\left(Q_{1}, \ldots, Q_{n}\right)$ of $n$ points in $\mathbb{R}^{2}$ is said to be

- in special position $(v 1)$ if $\mathrm{ev}^{-1}(Q)$ is infinite;

- in special position (v2) if $\mathrm{ev}^{-1}(Q)$ is infinite or intersects the codimension-1 skeleton of $\mathcal{M}_{0, n}^{\mathrm{lab}}\left(\mathbb{R}^{2}, \Delta\right)$.

Otherwise we say that $Q$ is in general position (for (v1) or (v2)). As ev is linear on each cone of $\mathcal{M}_{0, n}^{\mathrm{lab}}\left(\mathbb{R}^{2}, \Delta\right)$, note that $\mathrm{ev}^{-1}(Q)$ being infinite is equivalent to saying that the map ev is not injective on (at least) one cone of $\mathcal{M}_{0, n}^{\text {lab }}\left(\mathbb{R}^{2}, \Delta\right)$ that intersects $\operatorname{ev}^{-1}(Q)$.

Remark 3.6. Version (v2) is typically used for enumerative purposes: fix a point configuration $Q$ of points in general position (v2) and consider curves $C$ in $\mathrm{ev}^{-1}(Q)$, which contain only 3 -valent vertices. We assign a multiplicity mult $(C)$ to each curve $C$ (see for example $[\mathrm{GKM}])$. It can then be shown that $\sum_{C \in \mathrm{ev}^{-1}(Q)} \operatorname{mult}(C)$ is independent of the choice of $Q$; it is an invariant. 
Remark 3.7 (Welschinger invariants as intersection products). Inspired by $[\mathrm{MR}]$, one could hope to write real enumerative invariants, for example Welschinger invariants, as intersection products of adequate cycles in $\mathcal{M}_{0, n}^{\text {lab }}\left(\mathbb{R}^{2}, \Delta\right)$. Contrarily to the classical situation, the part of $\mathcal{M}_{0, n}^{\mathrm{lab}}\left(\mathbb{R}^{2}, \Delta\right)$ corresponding to real $n$-marked rational projective curves has the same dimension as $\mathcal{M}_{0, n}^{\text {lab }}\left(\mathbb{R}^{2}, \Delta\right)$. Hence, in order to impose some incidence conditions to these curves (like point, line or Psi-conditions) we would have to intersect a cycle of codimension 0 in $\mathcal{M}_{0, n}^{\text {lab }}\left(\mathbb{R}^{2}, \Delta\right)$ with pull-backs of rational functions cutting out points, lines, or Psi-classes. For real curves, this cycle should be different from $\mathcal{M}_{0, n}^{\text {lab }}\left(\mathbb{R}^{2}, \Delta\right)$, which corresponds to the case of complex curves. But Proposition 2.26 states that such a cycle cannot exist.

We now want to derive formulas for the locus in $\mathbb{R}^{2 n}$ of points in special position, for both versions (v1) and (v2).

Remark 3.8 (Strings). A parametrized tropical curve has a string if the underlying graph $\Gamma$ contains a subgraph homeomorphic to $\mathbb{R}$ which does not intersect the closures $\overline{x_{i}}$ of the markings $x_{1}, \ldots, x_{n}$. By [GM2, Remark 3.7] curves lying in codimension 0 of $\mathcal{M}_{0, n}^{\text {lab }}\left(\mathbb{R}^{2}, \Delta\right)$ passing through points in special position have at least one string. Such a curve can have several strings which are not necessarily disjoint.

Definition 3.9 (Free and fixed edges). Let $(C, h)$ with $C=\left(\Gamma ; x_{1}, \ldots, x_{n}\right)$ be a parametrized tropical curve in $\mathbb{R}^{2}$. Let $V$ be a vertex of $C$ and $E$ an adjacent edge. Then $E$ is called a free edge at $V$ if it can be connected in $\Gamma \backslash\left(V \cup \bigcup_{i=1}^{n} \overline{x_{i}}\right)$ to an unmarked end. Otherwise we call $E$ a fixed edge at $V$.

Proposition 3.10 (Points in special position (v1)). Let $n=|\Delta|-1$ and assume $n>1$. Then the set of points in special position (v1) for curves in $\mathcal{M}_{0, n}^{\mathrm{lab}}\left(\mathbb{R}^{2}, \Delta\right)$ equals the support of the cycle $\mathrm{ev}_{*}\left(\psi_{1}+\cdots+\psi_{n}\right)$.

Proof. We have to show two inclusions.

In order to prove that the set of points in special position is contained in the support of $\operatorname{ev}_{*}\left(\psi_{1}+\cdots+\psi_{n}\right)$ we consider curves lying in codimension 0 of $\mathcal{M}_{0, n}^{\text {lab }}\left(\mathbb{R}^{2}, \Delta\right)$ passing through points in special position. By Remark 3.8 these curves have at least one string. The idea of the proof is that moving such a string yields a curve passing through the same point configuration, but lying in a codimension-one cone of $\mathcal{M}_{0, n}^{\text {lab }}\left(\mathbb{R}^{2}, \Delta\right)$ and having one 4 -valent vertex where one of the adjacent edges is a marking $x_{1}, \ldots, x_{n}$. (Remember that a marking $x_{i}$ is an edge of the underlying abstract tropical curve that is mapped to a point in the parametrized curve, it is a contracted end. So a marking $x_{i}$ adjacent to a vertex is depicted by a point on the vertex below.) This cone is often not unique as we might move the string in different directions. Moreover, the resulting curve does not necessarily lie in the boundary of the original cone: it might happen that the string first runs into a 4 -valent vertex such that no marking $x_{i}$ is adjacent to it. When resolving this vertex a new string appears which can be moved again. The following sequence of pictures shows the idea of these movements; there is one string which is drawn in dashed lines. In the second picture there is a 4 -valent vertex without adjacent marking. The final curve has a 
marking at a 4 -valent vertex $x_{i}$, so it lies in the support of $\psi_{i}$, which means that our set of points in special position lies in the support of $\mathrm{ev}_{*} \psi_{i}$ and thus also of $\mathrm{ev}_{*}\left(\psi_{1}+\cdots+\psi_{n}\right)$.

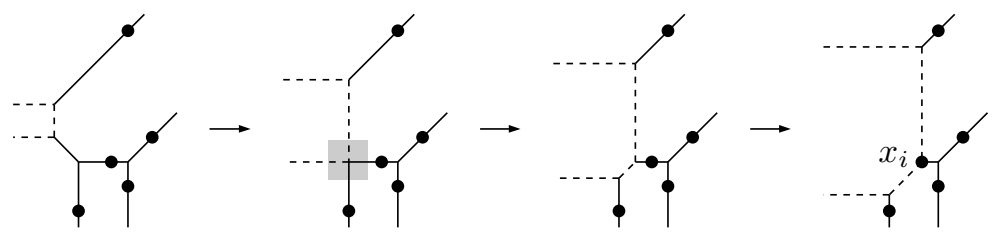

To make this argument rigorous we have to give an algorithm how to move a string so that it runs into a marking. For this let us first consider a curve with a 4 -valent vertex on a string without adjacent marking (as in the second picture above). The following picture shows the types of 4 -valent vertices without marking, where the types distinguish which of the adjacent edges are parallel.
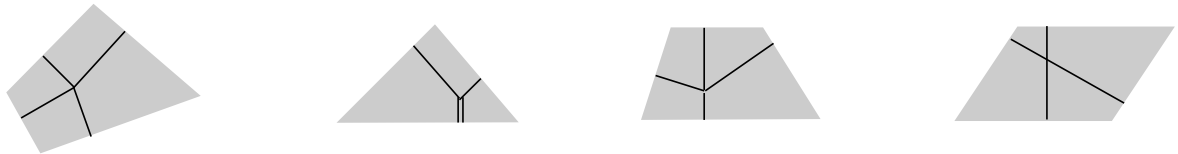

Note that at most two of the adjacent edges are fixed by the point conditions since at least two of them lie on a string. If none of the adjacent edges is fixed, the 4-valent vertex arises from the string movement in curves in codimension 0 having at least two strings joining in codimension 1 at the 4 -valent vertex. In this case, it is possible to move one of the strings differently in order to obtain a 4-valent vertex with at least one adjacent fixed edge. Let us assume this in the following. Then, considering all possibilities which of the edges can be fixed in each of the above types, one can see that in each case there is a resolution of the 4-valent vertex such that at least one of the fixed adjacent edges becomes shorter. For instance, for the first 4-valent vertex from the left in the picture above the table below lists the resolution(s) (A), (B) or (C), where at least one of the fixed edges a, b, c or d gets shorter, depending on which of the adjacent edges are fixed. Note that, in the case of one fixed edge, this edge becomes shorter in each of the resolutions.

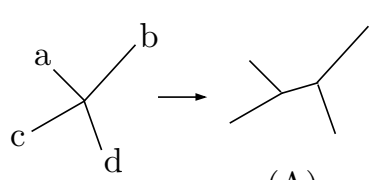

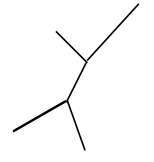

(B)

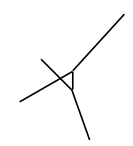

(C)

\begin{tabular}{|c||c|c|c|c|c|c|}
\hline edges fixed & a, b & a, c & a, d & b, c & b, d & c, d \\
\hline \hline resolutions & $(\mathrm{A}),(\mathrm{C})$ & $(\mathrm{B})$ & $(\mathrm{A}),(\mathrm{B})$ & $(\mathrm{A}),(\mathrm{B})$ & $(\mathrm{B}),(\mathrm{C})$ & $(\mathrm{A}),(\mathrm{C})$ \\
\hline
\end{tabular}

The algorithm now works as follows. Start with a triple $(C, S, E)$ where $C$ is the original curve, $S$ a string on $C$, and $E$ a fixed edge adjacent to $S$ (such a choice is possible since there is at least one marking). Denote by $M=M(C, S, E)$ the maximum distance in $C$ from $S$ to a marking behind $E$ which can be reached from $S$ without passing other markings. The following picture on the left shows such a choice; $S$ is again drawn with dashed lines, and $M$ is the length of the two line segments drawn in bold. 

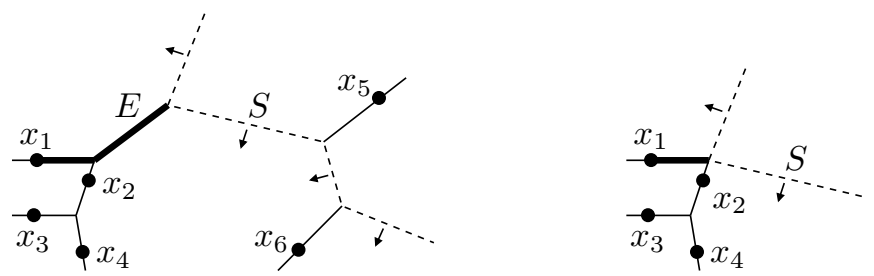

We now claim that we can always change the curve by moving the string (as indicated by the arrows) so that $M$ decreases - until either $M$ becomes zero and thus the string runs into a marking ( $x_{1}$ in the picture above), or the string runs into another marking elsewhere earlier (maybe $x_{6}$ in the picture above). The possibility of such a movement is obvious as long as the length of $E$ is positive. If $E$ shrinks to a point in the movement (as in the picture above on the right) we have a 4 -valent vertex at the string with at least one fixed adjacent edge, and by our above argument we know that we can always continue to move the string so that at least one of the fixed adjacent edges becomes shorter. Choosing this edge to be $E$ we can thus continue to decrease $M$ (note that by changing $E$ the set of first markings behind $E$ is replaced by a smaller one, so this step cannot make the maximum $M$ of their distances to $S$ bigger). This completes the argument and yields the first inclusion of the proposition.

For the other direction, we have to show that $\mathrm{ev}_{*}\left(\psi_{1}+\cdots+\psi_{n}\right)$ contains no points in general position. As $\sum_{i=1}^{n} \psi_{i}$ is a divisor, we just have to consider curves lying in codimension one of $\mathcal{M}_{0, n}^{\text {lab }}\left(\mathbb{R}^{2}, \Delta\right)$. So consider such a curve $C=\left(\Gamma ; x_{1}, \ldots, x_{n}\right)$ in $\psi_{i}$, i.e. a curve with one 4 -valent vertex $V$ with an adjacent marking $x_{i}$ and only 3 -valent vertices otherwise. We have to prove that we can deform $C$ to a codimension- 0 curve that still satisfies the same point conditions.

This is obvious if $C$ contains a string, so let us assume that this is not the case. Note that removing $\overline{x_{i}}$ from $\Gamma$ separates $\Gamma$ into 3 parts, whereas removing each of the other $n-1=|\Delta|-2$ causes one more separation. So $\Gamma \backslash \bigcup_{j=1}^{n} \overline{x_{j}}$ consists of $|\Delta|+1$ connected components. As none of these components can have more than one end (otherwise we would have a string) we conclude that there is precisely one bounded component with no end, whereas all other $|\Delta|$ components contain exactly one end. This means that at $V$ (which has $\psi_{i}$ and three more edges adjacent to it) at least two of the unmarked adjacent edges must be connected in $\Gamma \backslash \bigcup_{j=1}^{n} \overline{x_{j}}$ to an unbounded edge. We can then resolve $V$ so that these two edges $E_{1}$ and $E_{2}$ remain together but separate from $x_{i}$, forming a string and thus a movement of the curve with the positions of the markings fixed.

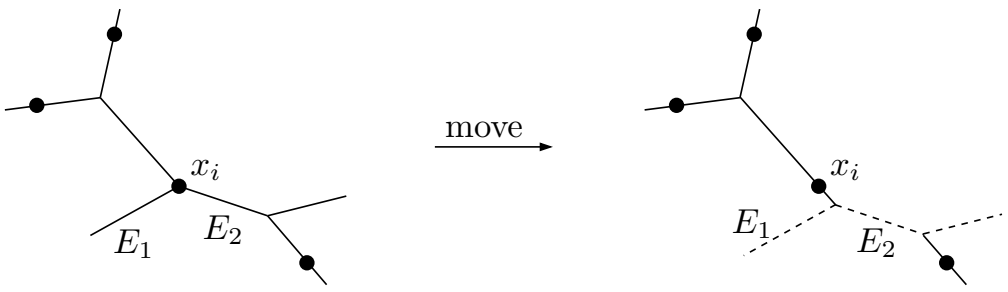

Hence the points are by definition in special position. 
Corollary 3.11. In the same situation as above, the set of points in special position (v1) for curves in $\mathcal{M}_{0, n}^{\mathrm{lab}}\left(\mathbb{R}^{2}, \Delta\right)$ can be given the structure of a tropical subfan in $\mathbb{R}^{2 n}$ of codimension one.

Proof. Observe that ev is a morphism of fans of dimension $2 n$. The claim then follows directly from Proposition 3.10 and Definition 3.3 (a) and (c).

Remark 3.12 (Comparison to the classical situation). As stated in the introduction of this chapter, the set of $n$ points in special position (v1) in the corresponding classical situation is a subvariety of $\left(\mathbb{P}^{2}\right)^{n}$. But in contrast to Corollary 3.11 it is not necessarily a subvariety of codimension one: consider for instance conics in $\mathbb{P}^{2}$ through 5 points. There are infinitely many conics through these points if and only if two of them coincide or four of them lie on a line - and this forms a subvariety of codimension 2 in $\left(\mathbb{P}^{2}\right)^{5}$.

The reason for the bigger dimension on the tropical side is that there are infinitely many liftings in the sense of $[\mathrm{JMM}]$ to $\mathbb{P}^{2}$ of the points in $\mathbb{R}^{2}$. When tropicalizing, the algebraic curves through each such configuration in $\left(\mathbb{P}^{2}\right)^{n}$ give rise to tropical curves passing through the given configuration of points in $\left(\mathbb{R}^{2}\right)^{n}$. As a consequence, the number of such tropical curves through the given points can be infinite although the number of algebraic curves through any lifting of them is not, i.e. the point configuration in $\left(\mathbb{R}^{2}\right)^{n}$ can be in special position (v1) although their liftings are not in special position classically.

On the other hand, returning to the case of conics, the locus of points where we find reducible curves through them (which roughly corresponds to (v2)) is the image of the locus of reducible curves in $\overline{\mathcal{M}}_{0,5}\left(\mathbb{P}^{2}, 2 \ell\right)$ under the evaluation map, where $\ell$ is the homology class of a line. Here, both this locus in $\overline{\mathcal{M}}_{0,5}\left(\mathbb{P}^{2}, 2 \ell\right)$ and its image in $\left(\mathbb{P}^{2}\right)^{5}$ has codimension 1 , the latter being the space of all points where three of them lie on a line.

Remark 3.13 (Generalization to curves with Psi-class conditions). One can generalize the result of Proposition 3.10 as follows to the case of counting curves satisfying Psi-class conditions (i.e to tropical descendant Gromov-Witten invariants) as in [MR]: fix $n>0$, a degree $\Delta$, and $k_{1}, \ldots, k_{n} \geqslant 0$ such that $n=|\Delta|-1-k_{1}-\cdots-k_{n}$. If we then modify Definitions 3.4 and 3.5 so that they use the moduli space $\psi_{1}^{k_{1}} \cdot \cdots \cdot \psi_{n}^{k_{n}} \cdot \mathcal{M}_{0, n}^{\mathrm{lab}}\left(\mathbb{R}^{2}, \Delta\right)$ instead of $\mathcal{M}_{0, n}^{\text {lab }}\left(\mathbb{R}^{2}, \Delta\right)$ we count curves through given points in $\mathbb{R}^{2}$ with additional Psiclass conditions, i.e. such that the valence of the vertex with attached marking $x_{i}$ is (at least) $k_{i}+3$ for all $i[\mathrm{KM}$, Theorem 4.1]. The proof of Proposition 3.10 can then easily be adapted to show that the set of points in special position (v1) equals the support of the cycle $\mathrm{ev}_{*}\left(\psi_{1}^{k_{1}} \cdot \cdots \cdot \psi_{n}^{k_{n}} \cdot\left(\psi_{1}+\cdots+\psi_{n}\right)\right)$. In fact, the first direction in the proof of the proposition remains unchanged since it is still true that curves passing through points in special position contain a string. In the second direction the curves in question will still contain exactly one bounded region in $\Gamma \backslash \bigcup_{j=1}^{n} \overline{x_{j}}$; it follows that the required movement of the curve is still possible, now resolving a $\left(k_{i}+4\right)$-valent vertex to a $\left(k_{i}+3\right)$-valent (containing the marking) and a 3 -valent vertex.

Proposition 3.14 (Points in special position (v2)). Fix $\Delta$ and let $n$ equal $|\Delta|-1$. Then the set of points in special position (v2) for curves in $\mathcal{M}_{0, n}^{\text {lab }}\left(\mathbb{R}^{2}, \Delta\right)$ equals the support of the push-forward $\mathrm{ev}_{*}\left(\mathcal{M}_{0, n}^{\mathrm{lab}}\left(\mathbb{R}^{2}, \Delta\right)_{\mathrm{sk}}^{(2 n-1)}\right)$ of the codimension-one skeleton of $\mathcal{M}_{0, n}^{\mathrm{lab}}\left(\mathbb{R}^{2}, \Delta\right)$ 
(note that this codimension-one skeleton is a cycle by Proposition 2.13 and Construction 3.2).

Proof. By definition the support of the cycle $\mathrm{ev}_{*}\left(\mathcal{M}_{0, n}^{\mathrm{lab}}\left(\mathbb{R}^{2}, \Delta\right)_{\mathrm{sk}}^{(2 n-1)}\right)$ contains only points in special position. In the other direction, if the points are in special position we can use the same argument as in the proof of Proposition 3.10 to show that they lie in the push-forward of the codimension-one skeleton of $\mathcal{M}_{0, n}^{\mathrm{lab}}\left(\mathbb{R}^{2}, \Delta\right)$.

Corollary 3.15. In the same situation as above, the set of points in special position (v2) for curves in $\mathcal{M}_{0, n}^{\mathrm{lab}}\left(\mathbb{R}^{2}, \Delta\right)$ can be given the structure of a tropical subfan in $\mathbb{R}^{2 n}$ of codimension one.

Proof. Observe that ev is a morphism of tropical fans of dimension $2 n$. The claim thus follows directly from Proposition 3.14 and Definition 3.3 (c).

Remark 3.16 (Reducible cycles). Using the characterization of Proposition 3.10, the set of points in special position (v1) for curves in $\mathcal{M}_{0, n}^{\text {lab }}\left(\mathbb{R}^{2}, \Delta\right)$ cannot be an irreducible cycle of $\mathbb{R}^{2 n}$ as it is the push-forward of the reducible divisor $\psi_{1}+\cdots+\psi_{n}$. Likewise, the set of points in special position (v2) for curves in $\mathcal{M}_{0, n}^{\text {lab }}\left(\mathbb{R}^{2}, \Delta\right)$ is not an irreducible cycle as Example 3.18 shows.

Remark 3.17 (Psi-condition). As in Remark 3.13 we can easily generalize Proposition 3.14 to the case of curves satisfying a Psi-condition $\psi_{i}$ in addition to incidence conditions with points; the result is then that the set of points in special position is the pushforward by ev of the codimension-one skeleton of $\psi_{i}$ (which is a cycle by Proposition 2.14). However, for more than one Psi-condition there is no similar statement as the codimension-one skeleton of a product of Psi-classes does not have a canonical choice of weights.

Example 3.18. Consider $\mathcal{M}_{0,2}^{\text {lab }}\left(\mathbb{R}^{2}, \Delta\right)$ with $\Delta=\left\{e_{1}+e_{2},-e_{1},-e_{2}\right\}$ where $e_{i}$ is the $i$-th standard basis vector of $\mathbb{R}^{2}$ (i.e. curves of degree one in the notation of [GKM, Definition 4.1]). Then $\mathcal{M}_{0,2}^{\text {lab }}\left(\mathbb{R}^{2}, \Delta\right)=\mathcal{M}_{0,5} \times \mathbb{R}^{2}$ by Construction 3.2. The space $\mathcal{M}_{0,5}$ can be represented by the Petersen graph, depicted below twice. The Petersen graph is a nonplanar, connected graph with 10 vertices denoted here $i j$ with $i, j \in\{1, \ldots, 5\}$ and $i<j$, which are connected by 15 edges s.t. a vertex $i j$ is only linked to the three vertices $k l$ with $k, l \in\{1, \ldots, 5\} \backslash\{i, j\}$. In this graph, the two-dimensional cones of $\mathcal{M}_{0,5}$ appear as edges, and the one-dimensional cones as vertices. The vertex $i j$ corresponds to the ray of $\mathcal{M}_{0,5}$ generated by the vector $v(\{i, j\})$.

$\mathcal{M}_{0,2}^{\text {lab }}\left(\mathbb{R}^{2}, \Delta\right)$ is particularly interesting as in this case the sets of points in special position of the two versions coincide, more precisely they even coincide as tropical fans with the weights of Corollaries 3.11 and 3.15. To see this, we observe that the codimensionone skeleton of $\mathcal{M}_{0,5}$ contains the cone 12 which is not contained in $\psi_{1}+\psi_{2}$. Furthermore, the cones 34, 35, and 45 have each weight 2 in the fan $\psi_{1}+\psi_{2}$ but just weight 1 each in the codimension-one skeleton of $\mathcal{M}_{0,5}$. But these cones 12, 34, 35, 45 vanish when pushed forward by ev, since ev is not injective on them (in fact their images consist of the configurations of two equal points in $\mathbb{R}^{2}$ and thus have codimension 2 in $\mathbb{R}^{2} \times \mathbb{R}^{2}$ ).

For more markings, the notions (v1) and (v2) will in general differ. 

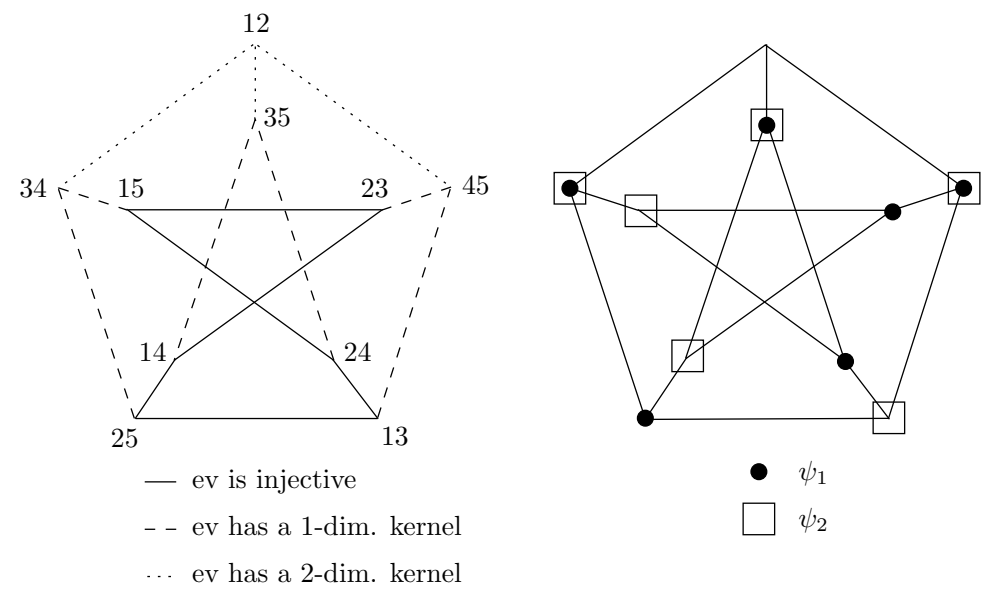

$\circ$

To be able to interpret Propositions 3.10 and 3.14 numerically we now want to compute the weights of the cells of codimension- 1 cycles in $\mathbb{R}^{2 n}$ that are of the form $\mathrm{ev}_{*}(Z)$ for a cycle $Z$ in $\mathcal{M}_{0, n}^{\text {lab }}\left(\mathbb{R}^{2}, \Delta\right)$. For this we first need to recall some well-known linear algebra results on elementary divisors.

Lemma and Definition 3.19 (Theorem on elementary divisors). Let $M$ be a finitely generated free module over a principal ideal domain $R$, and $N \subset M$ a submodule of $M$. Then there exists a basis $\left\{u_{1}, \ldots, u_{m}\right\}$ of $M$, a basis $\left\{v_{1}, \ldots, v_{n}\right\}$ of $N$ and $e_{1}, \ldots, e_{n} \in$ $R \backslash\{0\}$ such that $v_{i}=u_{i} e_{i}$ for $i=1, \ldots, n$ and $e_{i+1} \equiv 0 \bmod e_{i}$ for $i=1, \ldots, n-1$. The $e_{i}$ are called the elementary divisors of $N$ and are unique up to units in $R$.

For the quotient module it follows that $M / N \cong R^{m-n} \oplus \bigoplus_{i=1}^{n} R / R e_{i}$. Hence in the case $R=\mathbb{Z}$ the number of elements of the torsion part of $M / N$ is $\left|\prod_{i=1}^{n} e_{i}\right|$. In the following we will denote this number by $D(M / N)$.

Proof. See for example [L, Theorem III.7.8].

In the rest of the paper we will always use this result for the ring $R=\mathbb{Z}$. We then choose the $e_{i}$ to be positive.

Lemma 3.20. In the situation of Lemma 3.19 (for $R=\mathbb{Z}$ ) the number $D(M / N)$ is the greatest common divisor ( $g c d)$ of the $n \times n$ minors of any matrix $A$ representing the $\mathbb{Z}$-linear map $N \hookrightarrow M$.

We therefore denote this number by $D(A)$.

Proof. See Remark 3 of Chapter 12.2 on page 6 of [vdW]. From Lemma 3.19 it follows that the map $N \hookrightarrow M$ can be represented by a matrix $B$ with the elementary divisors on the diagonal and all other entries zero. As $A$ represents the same map there exist matrices $S \in \mathrm{GL}(m, \mathbb{Z})$ and $T \in \mathrm{GL}(n, \mathbb{Z})$ such that $A=S B T$. The $n \times n$ minors of $A$ are then integer linear functions of the $n \times n$ minors of $B$, and vice versa. As the gcd of the $n \times n$ minors of $B$ equals the product $D(M / N)=\prod_{i=1}^{n} e_{i}$, this means that, up to units in $\mathbb{Z}$, the ged of the $n \times n$ minors of $A$ equals $D(M / N)$ as well. 
Corollary 3.21. Let $B \in \mathbb{Z}^{(n+1) \times n}$ be a matrix having a $(n-k) \times(n-k)$ part $B_{1}$ in the upper left corner, a block $B_{2}$ of dimensions $(k+1) \times k$ in the lower right corner, an arbitrary (non-quadratic) block in the upper right corner, and just zeros in the lower left corner:

$$
B=\left(\begin{array}{c|c}
B_{1} & * \\
\hline 0 & B_{2}
\end{array}\right) .
$$

Then

$$
D(B)=\left|\operatorname{det}\left(B_{1}\right)\right| \cdot D\left(B_{2}\right) .
$$

Proof. To compute the $n \times n$ minors of $B$, we have to erase one row of $B$ and look at the determinants of these matrices. If we delete one of the first $n-k$ rows, the vectors in the columns of the quadratic part that remain are linearly dependent, hence these minors vanish. Deleting the $j$-th row with $n-k+1 \leqslant j \leqslant n+1$, we obtain a matrix that contains the block $B_{1}$ in the upper left corner, a quadratic block $C_{j}$ of dimensions $k \times k$ in the lower right corner and 0 in the lower left part. Hence the determinant of such a matrix equals the product of $\operatorname{det}\left(B_{1}\right)$ and $\operatorname{det}\left(C_{j}\right)$. So by Lemma 3.20 we get

$$
\begin{aligned}
D(B) & =\operatorname{gcd}\left\{\operatorname{det}\left(B_{1}\right) \cdot \operatorname{det}\left(C_{j}\right): j=n-k+1, \ldots, n+1\right\} \\
& =\left|\operatorname{det}\left(B_{1}\right)\right| \cdot \operatorname{gcd}\left\{\operatorname{det}\left(C_{j}\right): j=n-k+1, \ldots, n+1\right\} \\
& =\left|\operatorname{det}\left(B_{1}\right)\right| \cdot D\left(B_{2}\right),
\end{aligned}
$$

using the property $\operatorname{gcd}(m a, m b)=m \operatorname{gcd}(a, b)$ for $m \in \mathbb{N}$ and $a, b \in \mathbb{Z}$.

We will now apply these results to obtain formulas for the weights of push-forwards of codimension-1 cycles along the evaluation map. For this we first have to classify those cycles.

Remark 3.22 (Codimension-1 types in $\mathcal{M}_{0, n}^{\mathrm{lab}}\left(\mathbb{R}^{2}, \Delta\right)$ ). In the following, a connected component of $\Gamma \backslash \bigcup_{j=1}^{n} \overline{x_{j}}$ will be called a region of the curve. Consider a cell of a codimension-1 cycle in $\mathcal{M}_{0, n}^{\text {lab }}\left(\mathbb{R}^{2}, \Delta\right)$ on which the evaluation map is injective. It corresponds to a combinatorial type of curves having exactly one 4-valent vertex, with all other vertices being 3-valent.

If this 4-valent vertex has an adjacent marking, an argument as in the proof of the second part of Proposition 3.10 shows that there is exactly one region that is bounded (by markings), whereas the others contain exactly one end. We will call this type (A); in the picture below the bounded region is drawn with dotted lines.

If the 4 -valent vertex has no adjacent marking it lies in a unique region. The same argument as above then shows that

- either all regions have exactly one end (type (B) below, with the region containing the 4 -valent vertex drawn with dotted lines); or

- the region with the 4-valent vertex has exactly two ends, there is one other bounded region, and all other regions have exactly one end (type $(\mathrm{C})$ below, where the bounded region is drawn with dotted lines). 


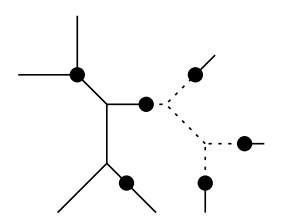

(A)

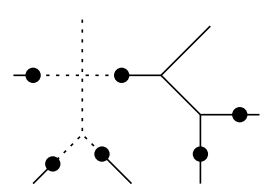

(B)

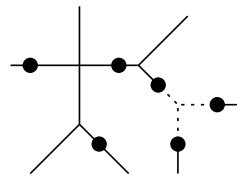

(C)

The weights of the images of those cycles under the evaluation map can be computed by the following formula.

Lemma and Definition 3.23. Let $Z$ be a cycle of dimension $2 n-1$ in $\mathcal{M}_{0, n}^{\mathrm{lab}}\left(\mathbb{R}^{2}, \Delta\right)$ and $\sigma^{\prime} \in \mathrm{ev}_{*}(Z) \subset \mathbb{R}^{2 n}$ a cone of the same dimension, i.e. of codimension 1 in $\mathbb{R}^{2 n}$. Then, with the convention and notations from Definition $3.3(c)$, the weight of $\sigma^{\prime}$ in the cycle $\mathrm{ev}_{*}(Z)$ is

$$
w_{\mathrm{ev}_{*}(Z)}\left(\sigma^{\prime}\right)=\sum_{\substack{\sigma \in Z \\ \operatorname{ev}(\sigma)=\sigma^{\prime}}} w_{Z}(\sigma) \cdot \operatorname{mult}(\sigma)
$$

where the multiplicity of a cone $\sigma$ of dimension $2 n-1$ in $\mathcal{M}_{0, n}^{\mathrm{lab}}\left(\mathbb{R}^{2}, \Delta\right)$ is defined as

$$
\operatorname{mult}(\sigma):=D\left(\mathbb{Z}^{2 n} / \mathrm{ev}\left(\Lambda_{\sigma}\right)\right) .
$$

If $C$ is any curve corresponding to a point in $\sigma$, we will write the multiplicity mult $(\sigma)$ also as $\operatorname{mult}(C)$.

Proof. By Definition 3.3 (c) we have to sum over the numbers $w_{Z}(\sigma) \cdot\left|\Lambda_{\sigma^{\prime}} / \operatorname{ev}\left(\Lambda_{\sigma}\right)\right|$ for all $\sigma \in Z$ with $\operatorname{ev}(\sigma)=\sigma^{\prime}$. But as $\Lambda_{\sigma^{\prime}}$ is by definition a saturated lattice in $\mathbb{Z}^{2 n} \subset \mathbb{R}^{2 n}$, we have $\mathbb{Z}^{2 n}=\Lambda_{\sigma^{\prime}} \oplus \mathbb{Z}$, and thus the torsion parts of $\Lambda_{\sigma^{\prime}} / \operatorname{ev}\left(\Lambda_{\sigma}\right)$ and $\mathbb{Z}^{2 n} / \operatorname{ev}\left(\Lambda_{\sigma}\right)$ agree.

Remark 3.24. By Lemma 3.19, the number mult $(\sigma)=D\left(\mathbb{Z}^{2 n} / \mathrm{ev}\left(\Lambda_{\sigma}\right)\right)$ in Lemma 3.23 can be computed as the $D(A)$ of any $(2 n) \times(2 n-1)$ matrix $A$ representing the $\mathbb{Z}$-linear map ev : $\Lambda_{\sigma} \rightarrow \mathbb{Z}^{2 n}$. By [GKM, Remark 5.2] and [GM2, Example 3.3] one possibility to set up this matrix is to use the lengths of all bounded edges and the position in $\mathbb{R}^{2}$ of a root vertex as coordinates for $\Lambda_{\sigma}$.

We will now compute explicitly the multiplicities in Lemma 3.23 for the cases of Remark 3.22 and proceed in several steps.

Lemma 3.25 (Splitting off vertex multiplicities). Let $C$ be a curve in a codimension-1 cone of $\mathcal{M}_{0, n}^{\mathrm{lab}}\left(\mathbb{R}^{2}, \Delta\right)$ as in Remark 3.22. Assume that there is an edge of $C$ such that splitting this edge yields two parts $C_{1}$ and $C_{2}$ of $C$, where $C_{2}$ contains only regions with exactly one end and having only 3-valent vertices. Then

$$
\operatorname{mult}(C)=\operatorname{mult}\left(C_{1}\right) \cdot \prod_{V \in C_{2}} \operatorname{mult}(V),
$$

where the product runs over all vertices in $C_{2}$ with no adjacent marking, and the multiplicity mult $(V)$ of such a vertex $V$ is defined as usual as the absolute value of the determinant of two of the adjacent direction vectors [GM2, Definition 3.5]. The following picture shows an example. 


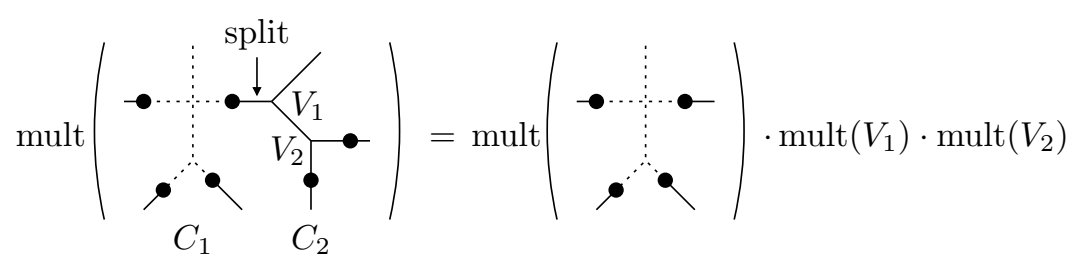

Proof. Let $k$ be the number of unbounded ends of $C_{2}$. Then $C_{2}$ has $k-1$ markings and $2 k-2$ bounded edges (including the split edge). Choosing the root vertex to be in $C_{1}$ (see Remark 3.24), only the $2 k-2$ coordinates of the $k-1$ markings in $C_{2}$ depend on the $2 k-2$ lengths of the bounded edges in $C_{2}$. Hence the matrix for ev as in Remark 3.24 has the form as in Corollary 3.21, with $B_{1}$ the $(2 k-2) \times(2 k-2)$ block consisting of these coordinates and lengths. As the absolute value of the determinant of $B_{1}$ equals the product of the vertex multiplicities in $C_{2}$ by [GM2, Proposition 3.8] and $B_{2}$ is precisely the matrix for the evaluation map on $C_{1}$, the claim follows from Corollary 3.21.

Lemma 3.26 (Multiplicity of a bounded region). Let $C$ be a 3-valent curve that has exactly one bounded region, with all other regions being single ends (such a curve occurs for instance as a part of the types (A) and (C) in Remark 3.22). Then the multiplicity of $C$ is

$$
\operatorname{mult}(C)=\operatorname{gcd}\{w(E): E \text { end in } C\} \cdot \prod_{V \in C} \operatorname{mult}(V),
$$

where the weight $w(E)$ of the edge $E$ is the gcd of the two coordinates of the direction vector of $E$, and the product is taken over all vertices of $C$ with no adjacent marking. The following picture shows an example.

$$
\operatorname{mult}\left(\begin{array}{ccc}
E_{1} & \sigma_{E_{2}} \\
V_{1} & \\
V_{2} & \bullet & E_{3} \\
& \bullet_{E_{4}}
\end{array}\right)=\operatorname{gcd}\left\{w\left(E_{1}\right), \ldots, w\left(E_{4}\right)\right\} \cdot \operatorname{mult}\left(V_{1}\right) \cdot \operatorname{mult}\left(V_{2}\right)
$$

Proof. We set up the matrix $A$ for the evaluation map as in Remark 3.24, with the root vertex within the bounded region. To compute the minors of $A$ as required by Lemma 3.20 we have to erase one of its rows. Note that the rows of $A$ correspond to the coordinates of the markings in $\mathbb{R}^{2}$. So let us assume that we erase the row for the $i$-th coordinate of the marking $x_{j}$ for some $i=1,2$ and $j=1, \ldots, n$. Note that the length of the bounded edge $E_{j}$ adjacent to $x_{j}$ is needed only for the coordinates of $x_{j}$ in $\mathbb{R}^{2}$, and so in the remaining matrix the column corresponding to $E_{j}$ has at most one non-zero entry, namely for the other coordinate of $x_{j}$ in $\mathbb{R}^{2}$. Laplace expansion of the determinant w.r.t. the $E_{j}$ column thus simply gives the product of this coordinate of $x_{j}$ in $\mathbb{R}^{2}$ and the determinant of the evaluation matrix for the curve where the marking $x_{j}$ is deleted (and thus $E_{j}$ becomes an unbounded end). But this determinant just equals the product of all vertex multiplicities by [GM2, Proposition 3.8].

Altogether we see that $D(A)$ is the product of all vertex multiplicities times the gcd of both coordinates of all markings in $\mathbb{R}^{2}$, as we have claimed. 
Corollary 3.27 (Multiplicity of the types $(\mathrm{A})$ and $(\mathrm{C})$ ). Let $C$ be a curve in codimension 1 as in Remark 3.22. If $C$ is of type (A) with bounded region $C_{b}$ then its multiplicity is

$$
\operatorname{mult}(C)=\operatorname{gcd}\left\{w(E): E \text { edge in } C_{b} \text { with adjacent marking }\right\} \cdot \prod_{V \in C} \operatorname{mult}(V) \text {. }
$$

If it is of type $(C)$ with bounded region $C_{b}$ then its multiplicity is

$$
\operatorname{mult}(C)=\operatorname{gcd}\left\{w(E): \text { E edge in } C_{b} \text { with adjacent marking }\right\} \cdot\left|\operatorname{det}\left(v, v^{\prime}\right)\right| \cdot \prod_{V \in C} \operatorname{mult}(V)
$$

where $v$ and $v^{\prime}$ are the directions of the two fixed adjacent edges at the 4-valent vertex (i.e. the ones that do not connect to an end within their region when coming from the 4-valent vertex). In both formulas, the product is taken over all 3-valent vertices without adjacent marking in $C$.

Proof. Let $V$ be the 4 -valent vertex of $C$. If $C$ is of type (A) we can first use Lemma 3.25 to split off all vertices behind the two unmarked edges adjacent to $V$ that do not lead to the bounded region. This way we get the multiplicities of all split-off vertices as a factor, and are left with a curve where two of the unmarked edges adjacent to $V$ are solitary ends (as it is already the case in the example picture in Remark 3.22). Now the evaluation matrix of this curve is precisely the same as for the curve where these two ends with direction vectors $v_{1}$ and $v_{2}$ are replaced by one end with direction $v_{1}+v_{2}$. Now in the remaining curve we can continue to split off all vertices that lie outside of the (closure of the) bounded region. This way we are left with a curve whose multiplicity has been computed in Lemma 3.26. Altogether, we get the result stated in the corollary.

If $C$ is of type (C) the procedure is very similar. We first split off all vertices behind the two free edges adjacent to $V$ and replace the resulting two solitary ends at $V$ by one. This makes the 4 -valent vertex $V$ into a new 3 -valent one for which two adjacent direction vectors are $v$ and $v^{\prime}$. As above, we continue to split off all vertices that are outside of the bounded region (one of which will be the new one with multiplicity $\left|\operatorname{det}\left(v, v^{\prime}\right)\right|$ ), and use Lemma 3.26 to obtain the result.

Lemma 3.28 (Multiplicity of a region with 4-valent vertex and one end). Let $C$ be $a$ curve in codimension 1 as in Remark 3.22 that has a region with a 4-valent vertex and one end directly adjacent to it, all other vertices being 3-valent and all other regions being single ends. Then the multiplicity of $C$ is

$$
\operatorname{mult}(C)=\operatorname{gcd}\left\{w(E) \cdot\left|\operatorname{det}\left(v_{E}^{1}, v_{E}^{2}\right)\right|: E \text { end with marking in } C\right\} \cdot \prod_{V \in C} \operatorname{mult}(V)
$$

where the product is taken over all 3-valent vertices of $C$ with no adjacent marking, and $v_{E}^{1}$ and $v_{E}^{2}$ denote the direction vectors of the two fixed edges adjacent to the 4-valent vertex that do not connect to $E$. The following picture shows an example. 


$$
\operatorname{mult}\left(\begin{array}{cc:c}
v_{1} & v_{2} \\
E_{1} & v & \bullet_{2} \\
E_{4} & \ddots & E_{3}
\end{array}\right)=\begin{array}{r}
\operatorname{gcd}\left\{w\left(E_{1}\right)\left|\operatorname{det}\left(v, v_{2}\right)\right|, w\left(E_{2}\right)\left|\operatorname{det}\left(v, v_{1}\right)\right|,\right. \\
\left.w\left(E_{3}\right)\left|\operatorname{det}\left(v_{1}, v_{2}\right)\right|, w\left(E_{4}\right)\left|\operatorname{det}\left(v_{1}, v_{2}\right)\right|\right\} \cdot \operatorname{mult}(V)
\end{array}
$$

Proof. The proof is very similar to that of Lemma 3.26. Let $V^{\prime}$ be the 4 -valent vertex of $C$ and $E$ its unique free end. We set up the matrix $A$ of the evaluation map using $V^{\prime}$ as the root vertex. To compute a maximal minor of $A$ we delete the row corresponding to the $i$-th coordinate of the marking $x_{j}$. Performing a Laplace expansion of the minor w.r.t. the column corresponding to the length of the bounded edge adjacent to $x_{j}$ we obtain the other coordinate of $x_{j}$ in $\mathbb{R}^{2}$ times the determinant of the evaluation matrix corresponding to the curve where the marking $x_{j}$ has been deleted. In this new curve we can use the technique of Lemma 3.25 to split off all vertices behind the one that lead to $x_{j}$. We can then replace the two resulting solitary ends at $V^{\prime}$ ( $E$ and the one just created by splitting off vertices) at $V^{\prime}$ by one, leading to a new 3 -valent vertex with multiplicity $\left|\operatorname{det}\left(v_{E}^{1}, v_{E}^{2}\right)\right|$. The resulting determinant gives the product of all vertex multiplicities by [GM2, Proposition 3.8]. Taking the gcd of these expressions for all rows of $A$ yields the desired result.

Corollary 3.29 (Multiplicity of the type (B)). Let $C$ be a curve in codimension 1 as in Remark 3.22. If $C$ is of type $(B)$, and $C^{\prime}$ denotes the region with the 4-valent vertex, then its multiplicity is

$$
\operatorname{mult}(C)=\operatorname{gcd}\left\{w(E) \cdot\left|\operatorname{det}\left(v_{E}^{1}, v_{E}^{2}\right)\right|\right\} \cdot \prod_{V \in C} \operatorname{mult}(V)
$$

where

- the product is taken over all 3-valent vertices without adjacent marking in $C$;

- the gcd is taken over all edges in $C^{\prime}$ that are adjacent to a marking and lie behind one of the three fixed edges adjacent to the 4-valent vertex; and

- $v_{E}^{1}$ and $v_{E}^{2}$ denote as in Lemma 3.28 the directions of the two fixed edges adjacent to the 4-valent vertex that do not connect to $E$.

Proof. The proof is similar to that of Corollary 3.27: first we can split off all vertices outside of $C^{\prime}$ and behind the one free end of the 4-valent vertex, and then we use Lemma 3.28 for the resulting curve.

Summarizing, we can now rephrase Lemma 3.23 as follows.

Corollary 3.30. Let $Z$ be a cycle of dimension $2 n-1$ in $\mathcal{M}_{0, n}^{\text {lab }}\left(\mathbb{R}^{2}, \Delta\right)$ and $\sigma^{\prime} \in \mathrm{ev}_{*}(Z) \subset$ $\mathbb{R}^{2 n}$ a cone of the same dimension, i.e. of codimension 1 in $\mathbb{R}^{2 n}$. Then, with the convention and notations from Definition 3.3 (c), the weight of $\sigma^{\prime}$ in the cycle $\mathrm{ev}_{*}(Z)$ is

$$
w_{\mathrm{ev}_{*}(Z)}\left(\sigma^{\prime}\right)=\sum_{\substack{\sigma \in Z \\ \operatorname{ev}(\sigma)=\sigma^{\prime}}} w_{Z}(\sigma) \cdot \operatorname{mult}(\sigma)
$$


where the multiplicity mult $(\sigma)$ of a cone $\sigma$ of dimension $2 n-1$ in $\mathcal{M}_{0, n}^{\mathrm{lab}}\left(\mathbb{R}^{2}, \Delta\right)$ is given by Corollaries 3.27 and 3.29 depending on their type as in Remark 3.22.

In particular, this gives an explicit formula for the cycles in $\mathbb{R}^{2 n}$ of points in special position (v1) and (v2) as in Propositions 3.10 and 3.14.

\section{References}

[AC $\quad$ E. Arbarello, M. Cornalba, Combinatorial and algebro-geometric cohomology classes on the moduli spaces of curves, J. Alg. Geom. 5 (1996), 705-749.

[AR] L. Allermann, J. Rau, First steps in tropical intersection theory, Math. Z. 264 (2010), 633-670.

[B] K. Behrend, Gromov-Witten invariants in algebraic geometry, Inv. Math. 127 (1997), no. 3, 601-617.

[BJSST] T. Bogart, A. Jensen, D. Speyer, B. Sturmfels, R. Thomas, Computing tropical varieties, J. Symb. Comput. 42 (2007), no. 1-2, 54-73.

[EGAIV] A. Grothendieck, Éléments de géométrie algébrique IV: Étude locale des schémas et des morphismes de schémas, Publ. Math. de l'IHÉS 28 (1966).

[FP] W. Fulton, R. Pandharipande, Notes on stable maps and quantum cohomology, Proc. Symp. Pure Math. 62 (1997) part 2, 45-96.

[GM1] A. Gathmann, H. Markwig, The numbers of tropical plane curves through points in general position, J. Reine Angew. Math. 602 (2007), 155-177.

[GM2] A. Gathmann, H. Markwig, Kontsevich's formula and the WDVV equations in tropical geometry, Adv. in Math. 217 (2008), 537-560.

[GKM] A. Gathmann, M. Kerber, H. Markwig, Tropical fans and the moduli spaces of tropical curves, Compos. Math. 145 (2009), no. 1, 173-195.

[H] M. Herold, Intersection theory of the tropical moduli spaces of curves, Diploma thesis, TU Kaiserslautern (2007), https://kluedo.ub.uni-kl.de/frontdoor/index/index/docId/3276.

[JMM] A. N. Jensen, H. Markwig, T. Markwig, An algorithm for lifting points in a tropical variety, Collect. Math. 59 (2008), no. 2, 129-165.

[K] S. Keel, Intersection theory of moduli space of stable $N$-pointed curves of genus zero, Trans. Amer. Math. Soc. 330 (1992), no. 2, 545-574.

[KM] M. Kerber, H. Markwig, Intersecting Psi-classes on tropical $\mathcal{M}_{0, n}$, Int. Math. Res. Not. (2009), 221-240.

[K1] F. Knudsen, The projectivity of the moduli space of stable curves. II, Math. Scand. 52 (1983), 161-199.

[K2] F. Knudsen, The projectivity of the moduli space of stable curves. III, Math. Scand. 52 (1983), 200-212. 
[KoM] M. Kontsevich, Y. Manin, Relations between the correlators of the topological sigma-model coupled to gravity, Commun. Math. Phys. 196 (1998), 385-398.

[L] S. Lang, Algebra, Springer Graduate Texts in Mathematics, New York (2002).

[M] G. Mikhalkin, Moduli spaces of rational tropical curves, Proceedings of Gkova Geometry-Topology Conference GGT 2006 (2007), 39-51.

[MR] H. Markwig, J. Rau, Tropical descendant Gromov-Witten invariants, Manuscr. Math. 129 (2009), no. 3, 293-335.

[R] J. Rau, Intersections on tropical moduli spaces, preprint (2008).

[SS] D. Speyer, B. Sturmfels, The tropical Grassmannian, Adv. in Geom. 4 (2004), 389-411.

[vdW] B. van der Waerden, Algebra, Volume II, Springer, New York (1991). 